\title{
Antiferromagnetic spin structure and lithium ion diffusion in $\mathrm{Li}_{2} \mathrm{MnO}_{3}$ probed by $\mu^{+} \mathrm{SR}$
}

\author{
Jun Sugiyama,,${ }^{1,}$ Kazuhiko Mukai, ${ }^{1}$ Hiroshi Nozaki, ${ }^{1}$ Masashi Harada, ${ }^{1}$ Martin Månsson, ${ }^{2}$ Kazuya Kamazawa, ${ }^{3}$ \\ Daniel Andreica, ${ }^{4}$ Alex Amato, ${ }^{5}$ and Adrian D. Hillier ${ }^{6}$ \\ ${ }^{1}$ Toyota Central Research and Development Laboratories, Inc., Nagakute, Aichi 480-1192, Japan \\ ${ }^{2}$ Laboratory for Solid State Physics, ETH Zürich, CH-8093 Zürich, Switzerland \\ ${ }^{3}$ Comprehensive Research Organization for Science and Society, Tokai, Ibaragi 319-1106, Japan \\ ${ }^{4}$ Faculty of Physics, Babes-Bolyai University, 3400 Cluj-Napoca, Romania \\ ${ }^{5}$ Laboratory for Muon Spin Spectroscopy, Paul Scherrer Institut, CH-5232 Villigen PSI, Switzerland \\ ${ }^{6}$ ISIS Muon Facility, Rutherford Appleton Laboratory, Didcot, Oxfordshire, OX11 OQX, United Kingdom
}

(Received 23 August 2012; revised manuscript received 17 October 2012; published 14 January 2013)

\begin{abstract}
In order to elucidate the antiferromagnetic (AF) spin structure below $T_{\mathrm{N}} \sim 35 \mathrm{~K}$ and to clarify the diffusive behavior of $\mathrm{Li}^{+}$ions in the layered compound $\mathrm{Li}_{2} \mathrm{MnO}_{3}$, we have performed a muon-spin rotation and relaxation $\left(\mu^{+} \mathrm{SR}\right)$ experiment using a powder sample in the temperature range between 2 and $500 \mathrm{~K}$. Below $T_{\mathrm{N}}$, the zero-field (ZF-) $\mu^{+}$SR spectrum showed a clear oscillation that consists of two muon-spin precession signals with different frequencies. Combining with the dipole field calculations, it was found that the most probable spin structure for $\mathrm{Li}_{2} \mathrm{MnO}_{3}$ is the $C_{x}$-type AF order in which $\mathrm{Mn}$ moments align parallel or antiparallel to the $a$ axis in the $\left[\mathrm{Li}_{1 / 3} \mathrm{Mn}_{2 / 3}\right] \mathrm{O}_{2}$ layer, and a ferromagnetic chain along the $a$ axis aligns antiferromagnetically along both the $b$ and $c$ axes. The ordered Mn moment was estimated as $2.62 \mu_{\mathrm{B}}$ at $2 \mathrm{~K}$. In the paramagnetic state, ZF- and longitudinal-field $\mu^{+}$SR spectra exhibited a dynamic nuclear field relaxation. From the temperature dependence of the field distribution width, the $\mathrm{Li}^{+}$ions were found to diffuse mainly along the $c$ axis through the $\mathrm{Li}$ ion in the $\left[\mathrm{Li}_{1 / 3} \mathrm{Mn}_{2 / 3}\right] \mathrm{O}_{2}$ layer. Also, based on the field fluctuation rate, a self-diffusion coefficient of $\mathrm{Li}^{+}$ions $\left(D_{\mathrm{Li}}\right)$ at $300 \mathrm{~K}$ was estimated as $4.7(4) \times 10^{-11} \mathrm{~cm}^{2} / \mathrm{s}$ with the thermal activation energy $E_{a}=0.156(3) \mathrm{eV}$.
\end{abstract}

DOI: 10.1103/PhysRevB.87.024409

PACS number(s): 76.75.+i, 75.25.-j, 75.50.Ee, 66.30.H-

\section{INTRODUCTION}

Alkali transition-metal dioxides, $A M \mathrm{O}_{2}$, with an $\alpha$ $\mathrm{NaFeO}_{2}$-type structure were originally studied due to their complex magnetic nature caused by a competition between geometrical frustration and antiferromagnetic (AF) correlation on the two-dimensional triangular lattice of $M$ ions, ${ }^{1-3}$ which is formed by a network of edge sharing $M \mathrm{O}_{6}$ octahedra. Besides the strong interests in their magnetism from the viewpoint of condensed matter physics, $A M \mathrm{O}_{2}$, particularly $\mathrm{Li} M \mathrm{O}_{2}$, has been heavily investigated as a positive electrode material for Li-ion batteries due to a reversible extraction (insertion) reaction of $\mathrm{Li}^{+}$ions from (into) the lattice. ${ }^{4-7}$

The target compound, $\mathrm{Li}_{2} \mathrm{MnO}_{3}$, belongs to a monoclinic system with $C 2 / m$ space group, and $a=0.4937 \mathrm{~nm}, b=$ $0.8532 \mathrm{~nm}, c=0.5032 \mathrm{~nm}$, and $\beta=109.46^{\circ}$ at ambient temperature $(T)^{8}$ (see Fig. 1). Since the $\mathrm{Li}_{2} \mathrm{MnO}_{3}$ structure consists of alternating stack of a $\mathrm{Li}$ plane and $\left[\mathrm{Li}_{1 / 3} \mathrm{Mn}_{2 / 3}\right]_{2}$ layer along the $c$ axis, $\mathrm{Li}_{2} \mathrm{MnO}_{3}$ could be also classified as a layered $\mathrm{AMO}_{2}$.

Magnetically, $\mathrm{Li}_{2} \mathrm{MnO}_{3}$ is known to be an AF insulator with $T_{\mathrm{N}} \sim 50 \mathrm{~K} .{ }^{9}{ }^{, 10}$ Neutron diffraction measurements ${ }^{8}$ revealed that the AF unit cell is doubled along the $c$ axis. In addition, the AF spin structure was proposed as an $A$-type AF order, in which the Mn moments align ferromagnetically along the $c$ axis in the $\left[\mathrm{Li}_{1 / 3} \mathrm{Mn}_{2 / 3}\right] \mathrm{O}_{2}$ layer, but antiferromagnetically between the adjacent $\left[\mathrm{Li}_{1 / 3} \mathrm{Mn}_{2 / 3}\right] \mathrm{O}_{2}$ layers. However, since another structure was also proposed in Ref. 8, the AF spin structure is still not fully understood at present.

Electrochemically, $\mathrm{Li}_{2} \mathrm{MnO}_{3}$ was found to exhibit a reversible extraction-insertion reaction of $\mathrm{Li}^{+},{ }^{11}$ which led to an intensified effort to clarify the relationship between structural and/or physical parameters and electrochemical properties. ${ }^{12-18}$ Detailed electrochemical measurements combined with XRD analysis suggested the formation of oxygen deficiency during extraction-insertion reactions in order to keep charge neutrality. ${ }^{19,20}$ This was supported by recent first-principles calculations. ${ }^{21}$ As a result, the $\mathrm{Li}_{2} \mathrm{MnO}_{3}$ phase gradually transforms into a spinel-like phase during the extraction reaction. ${ }^{22}$ Furthermore, since a solid solution system between $\mathrm{Li}_{2} \mathrm{MnO}_{3}$ and $\mathrm{Li} M \mathrm{O}_{2}$ has a very high capacity compared with those of the conventional positive electrode materials, ${ }^{19}$ such " $\mathrm{Li}_{2} \mathrm{MnO}_{3}$-stabilized $\mathrm{LiMO} \mathrm{O}_{2}$ " compounds are extensively investigated as a positive electrode material for the future Li-ion batteries. ${ }^{23}$

Despite a main stream of research towards the battery application, the nature of $\mathrm{Li}_{2} \mathrm{MnO}_{3}$, particularly, a correct $\mathrm{AF}$ spin structure and a diffusive behavior of $\mathrm{Li}^{+}$ions, are still not clarified so far. If the $A$-type AF order is formed below $T_{\mathrm{N}}$, as in the case for $\mathrm{NaNiO}_{2},{ }^{24}$ the interaction between the neighboring $\mathrm{Mn}$ ions is naturally ferromagnetic (FM), and the interaction via an Mn-O-Li-O-Mn path is AF along the $c$ axis. However, such interaction should be FM or negligibly small in the $\left[\mathrm{Li}_{1 / 3} \mathrm{Mn}_{2 / 3}\right] \mathrm{O}_{2}$ layer. In order to further elucidate the magnetic nature of $\mathrm{Li}_{2} \mathrm{MnO}_{3}$, we have made a positive muon-spin rotation and relaxation $\left(\mu^{+} \mathrm{SR}\right)$ experiment, because $\mu^{+} \mathrm{SR}$ provide information on magnetic environment at the vicinity of implanted muons. Indeed, $\mu^{+} \mathrm{SR}$ clarified the magnetic nature of many $A M \mathrm{O}_{2}$ compounds, such as $\mathrm{LiCrO}_{2},{ }^{25,26} \mathrm{NaCrO}_{2},{ }^{27}$ $\mathrm{Li}_{x} \mathrm{CoO}_{2},{ }^{28,29} \mathrm{Na}_{x} \mathrm{CoO}_{2},{ }^{30-35} \mathrm{~K}_{x} \mathrm{CoO}_{2},{ }^{36,37} \mathrm{Li}_{x} \mathrm{NiO}_{2},{ }^{38,39}$ and $\mathrm{NaNiO}_{2} \cdot{ }^{40}$ Although preliminary results on $\mathrm{Li}_{2} \mathrm{MnO}_{3}$ were already described elsewhere, ${ }^{41,42}$ we definitely need more detailed analysis in order to determine the AF spin structure.

Concerning Li diffusion, since magnetic ions in the lattice strongly affect a Li-NMR signal even in a paramagnetic 


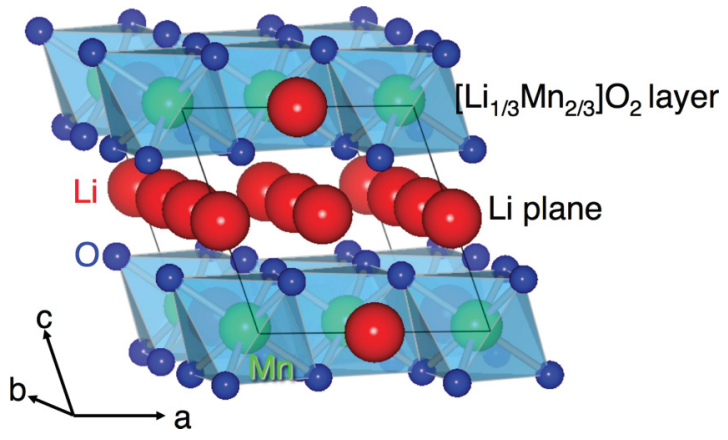

FIG. 1. (Color online) Crystal structure of $\mathrm{Li}_{2} \mathrm{MnO}_{3}$. Solid lines represent a crystallographic unit cell.

state of $\mathrm{Li}_{x} \mathrm{CoO}_{2},{ }^{43,44}$ it is extremely difficult to measure a spin-lattice relaxation rate $\left(1 / T_{1}\right)$ caused by $\mathrm{Li}$ diffusion. In fact, past $\mathrm{Li}-\mathrm{NMR}$ work on $\mathrm{Li}_{2} \mathrm{MnO}_{3}$ did not yield a self-diffusion coefficient of $\mathrm{Li}^{+}\left(D_{\mathrm{Li}}\right){ }^{45,46}$ Furthermore, due to a structural transformation during the $\mathrm{Li}^{+}$extraction reaction and a lack of information on the correct surface area between electrode and electrolyte, it is difficult to estimate $D_{\mathrm{Li}}$ for $\mathrm{Li}_{2} \mathrm{MnO}_{3}$ by electrochemical measurements. Hence, although $D_{\mathrm{Li}}$ is one of the most fundamental parameters to explain the rate of the extraction-insertion reaction, both Li-NMR and electrochemical measurements provide a very rough estimate for $D_{\mathrm{Li}}$.

On the contrary, $\mu^{+} \mathrm{SR}$ is found to give more reliable $D_{\mathrm{Li}}$ in $\mathrm{Li}_{x} \mathrm{CoO}_{2}{ }^{47}$ than $\mathrm{Li}-\mathrm{NMR},{ }^{44}$ where $\mathrm{Li}_{x} \mathrm{CoO}_{2}$ is known to be a common cathode material for Li-ion batteries, ${ }^{4}$ This is because muons do not feel fluctuating magnetic moments at high $T$, but instead sense the change in nuclear dipole field due to Li diffusion. Even if magnetic moments still affect the muon-spin depolarization rate, such an effect is, in principle, distinguishable from that of nuclear dipole fields. ${ }^{48}$ In particular, a weak longitudinal field (LF) can be applied that decouples the magnetic and nuclear dipole interactions. ${ }^{49}$ Here, LF means the magnetic field parallel to the initial muon-spin polarization. Following upon the initial attempt for measuring $D_{\mathrm{Li}}$ by $\mu^{+} \mathrm{SR},{ }^{47}$ the diffusive nature has been investigated for $\mathrm{LiNiO}_{2},{ }^{39} \mathrm{LiCrO}_{2},{ }^{39} \mathrm{Li}_{x}\left(\mathrm{Co}_{1 / 3} \mathrm{Ni}_{1 / 3} \mathrm{Mn}_{1 / 3}\right) \mathrm{O}_{2},{ }^{50}$ and $\mathrm{Li} M \mathrm{PO}_{4}$ with $M=\mathrm{Fe}, \mathrm{Co}, \mathrm{Ni} \cdot{ }^{51-53}$ Here, we report the $\mu^{+} \mathrm{SR}$ results of $\mathrm{Li}_{2} \mathrm{MnO}_{3}$ in order to clarify both the AF ground state at low $T$ and the diffusion path and $D_{\mathrm{Li}}$ at high $T$.

\section{EXPERIMENTAL}

A powder sample of $\mathrm{Li}_{2} \mathrm{MnO}_{3}$ was prepared by a solidstate reaction technique between $\mathrm{LiOH} \cdot \mathrm{H}_{2} \mathrm{O}$ and $\mathrm{MnOOH}$ $(\mathrm{Li} / \mathrm{Mn}=2.00 / 1.00)$ at $900{ }^{\circ} \mathrm{C}$ under air for $12 \mathrm{~h}$. According to a powder $\mathrm{X}$-ray diffraction (XRD) analysis, the sample was assigned as a single phase of a monoclinic symmetry with space group $C 2 / \mathrm{m}$.

In order to know the macroscopic magnetic properties of the sample, susceptibility $(\chi)$ was measured below $400 \mathrm{~K}$ under a $H \leqslant 10 \mathrm{kOe}$ field with a superconducting quantum interference device (SQUID) magnetometer (MPMS, Quantum Design). The Weiss temperature $\left(\Theta_{\mathrm{CW}}\right)$ and effective magnetic moment $\left(\mu_{\text {eff }}\right)$ were determined from the $\chi(T)$ curve by fitting to a Curie-Weiss law, $\chi=C /\left(T-\Theta_{\mathrm{CW}}\right)$ and
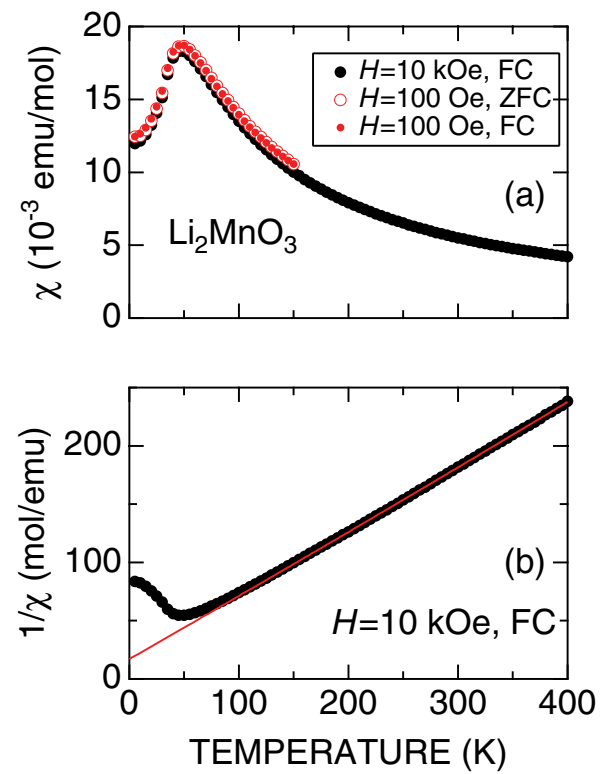

FIG. 2. (Color online) $T$ dependence of (a) susceptibility $(\chi)$ and (b) $1 / \chi$ for $\mathrm{Li}_{2} \mathrm{MnO}_{3}$. The $\chi$ data were obtained in field cooling (FC) mode with $H=10 \mathrm{kOe}$. In (a), the data obtained in FC and zero-field cooling (ZFC) mode with $H=100$ Oe are also plotted for comparison. In (b), the solid line represents a linear fit in the $T$ range between 200 and $400 \mathrm{~K}$ using a Curie-Weiss formula.

$C=\left(N / 3 k_{\mathrm{B}}\right) \mu_{\text {eff }}^{2}$ in the $T$ range between 200 and $400 \mathrm{~K}$. Here, $N$ is the density of $\mathrm{Mn}$ spins, $\mu_{\text {eff }}$ is the effective magnetic moment per $\mathrm{Mn}$, and $k_{\mathrm{B}}$ is Boltzmann's constant. As a result, we obtained $\Theta_{\mathrm{CW}}=-32 \pm 2 \mathrm{~K}$ and $\mu_{\text {eff }}=3.86 \pm 0.02 \mu_{\mathrm{B}}$, respectively (see Fig. 2). These values are similar to those for polycrystalline samples found in the literature. ${ }^{9,10}$

The $\mu^{+}$SR spectra were measured at the surface muon beam lines using the Dolly spectrometer of $\mathrm{S} \mu \mathrm{S} / \mathrm{PSI}$ in Switzerland and the MuSR spectrometer of ISIS/RAL in United Kingdom. At PSI, the approximatively 500-mg powder sample was placed in an envelope with $1 \mathrm{~cm} \times 1 \mathrm{~cm}$ area, which is made of Al-coated Mylar tape with $50-\mu \mathrm{m}$ thickness in order to minimize the signal from the envelope. The envelope was then attached to a low-background sample holder in a liquid-He flow-type cryostat in the temperature range between 1.8 and $250 \mathrm{~K}$. At ISIS, on the other hand, a $\sim 2 \mathrm{~g}$ powder sample was pressed into a disk with 23-mm diameter and 1-mm thickness and packed into an Au O-ring sealed titanium cell. The window of the cell was made of a Kapton film with $50-\mu \mathrm{m}$ thickness. The cell was mounted onto the Al plate of a He flow-type cryofurnace in the temperature range between 50 and $500 \mathrm{~K}$. The experimental techniques are described in more detail elsewhere. $^{54}$

\section{RESULTS}

\section{A. Nature of $\mathbf{M n}^{4+} \mathrm{AF}$ order at low temperature}

Figure 3(a) exhibits the $\mathrm{ZF}-\mu^{+} \mathrm{SR}$ spectrum for $\mathrm{Li}_{2} \mathrm{MnO}_{3}$ obtained at the lowest $T$ measured $(1.8 \mathrm{~K})$. One can clearly see the muon-spin precession signal with the frequency, $f_{\mathrm{H}} \sim$ $40 \mathrm{MHz}$, demonstrating the formation of static AF order. Also, the main precession signal is found to be slightly modulated by 

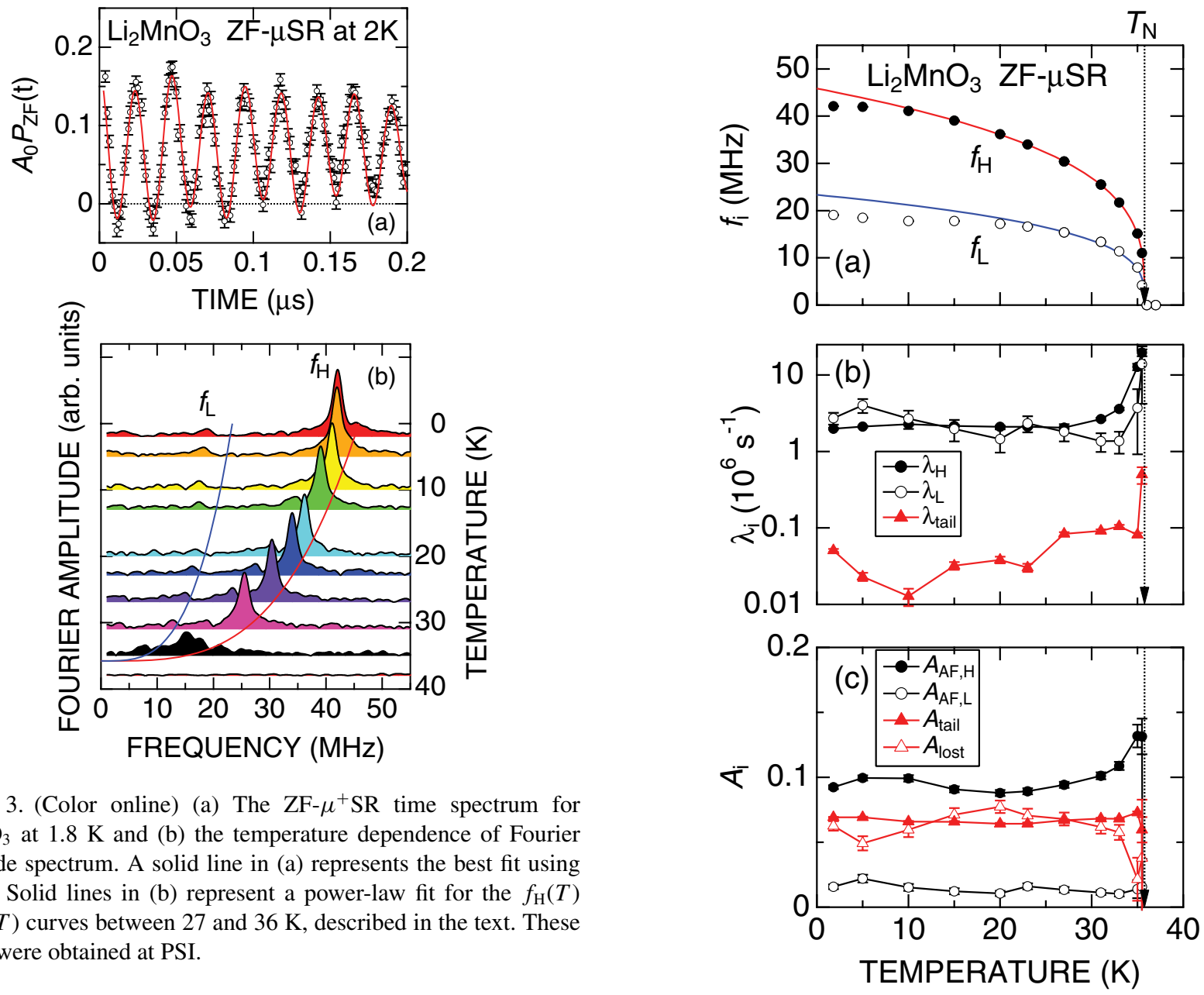

another precession signal, which we ignored in the previous report. ${ }^{42}$ The Fourier transform spectrum of the $\mathrm{ZF}-\mu^{+} \mathrm{SR}$ spectrum indicates that the second oscillatory signal has a frequency of $\sim 20 \mathrm{MHz}\left(=f_{\mathrm{L}}\right)$ at $1.8 \mathrm{~K}$ [see Fig. 3(b)]. Since the two frequencies decrease with $T$ and finally disappear at $T_{\mathrm{N}}$ $(\sim 35 \mathrm{~K})$, the result indicates the presence of two magnetically different muon sites in the lattice.

In fact, the ZF spectra were well fitted by a combination of two exponentially relaxing cosine oscillations for the static internal field and an exponentially relaxing nonoscillatory signal:

$$
\begin{aligned}
A_{0} P_{\mathrm{ZF}}(t)= & A_{\mathrm{AF}, \mathrm{H}} \cos \left(2 \pi f_{\mathrm{H}} t+\phi_{\mathrm{H}}\right) \exp \left(-\lambda_{\mathrm{H}} t\right) \\
& +A_{\mathrm{AF}, \mathrm{L}} \cos \left(2 \pi f_{\mathrm{L}} t+\phi_{\mathrm{L}}\right) \exp \left(-\lambda_{\mathrm{L}} t\right) \\
& +A_{\text {tail }} \exp \left(-\lambda_{\text {tail }} t\right),
\end{aligned}
$$

where $A_{0}$ is the initial asymmetry, $P_{\mathrm{ZF}}(t)$ is the muon spin polarization function under $\mathrm{ZF}, A_{\mathrm{AF}, \mathrm{H}}, A_{\mathrm{AF}, \mathrm{L}}$, and $A_{\text {tail }}$ are the asymmetries associated with the three signals. $f_{i}\left(\equiv \omega_{i}^{\mu} / 2 \pi\right)$ is the muon Larmor frequency corresponding to the static internal AF field, $\phi_{i}$ is the initial phase of the oscillatory signal, $\lambda_{i}$ and $\lambda_{\text {tail }}$ are the exponential relaxation rates of the three signals.

Figure 4 shows the $T$ dependencies of the $\mu^{+}$SR parameters obtained by fitting the ZF-spectra using Eq. (1). The $f_{\mathrm{H}}(T)$ and $f_{\mathrm{L}}(T)$ curves exhibit an order parameter like $T$ dependence, as expected. By using a power law, $f_{i} / f_{i}(0 \mathrm{~K})=\left[\left(T_{\mathrm{N}}^{\mathrm{ZF}}-\right.\right.$ $\left.T) / T_{\mathrm{N}}^{\mathrm{ZF}}\right]^{\beta}$, between 27 and $36 \mathrm{~K}$, which corresponds to $0.25 \geqslant\left(T_{\mathrm{N}}^{\mathrm{ZF}}-T\right) / T_{\mathrm{N}}$, the critical exponent $(\beta)$ is estimated

FIG. 4. (Color online) $T$ dependencies of the $\mu^{+}$SR parameters for $\mathrm{Li}_{2} \mathrm{MnO}_{3}$; (a) muon-spin precession frequencies $\left(f_{i}\right)$, (b) their relaxation rates $\left(\lambda_{i}\right)$ and the relaxation rate of nonoscillatory signal $\left(\lambda_{\text {tail }}\right)$, and (c) their asymmetries $\left(A_{i}\right.$ and $\left.A_{\text {tail }}\right)$ and a lost asymmetry $A_{\text {lost }}\left(=0.24-A_{\mathrm{AF}, \mathrm{H}}-A_{\mathrm{AF}, \mathrm{L}}-A_{\text {tail }}\right)$. The data were obtained by fitting the ZF spectra using Eq. (1). In (a), the solid lines on the $f_{i}(T)$ curve correspond to $f(T)=f(0 \mathrm{~K})\left(1-T / T_{\mathrm{N}}\right)^{\beta}$ with $f_{\mathrm{H}}(0 \mathrm{~K})=43.8 \pm 0.5 \mathrm{MHz}\left[f_{\mathrm{L}}(0 \mathrm{~K})=23.8 \pm 0.8 \mathrm{MHz}\right], T_{\mathrm{N}}=$ $35.768 \pm 0.002 \mathrm{~K}$, and $\beta=0.2916 \pm 0.0007$.

as $0.2916 \pm 0.0007, f_{\mathrm{H}}(0 \mathrm{~K})$ as $43.8 \pm 0.5 \mathrm{MHz}, f_{\mathrm{L}}(0 \mathrm{~K})$ as $23.8 \pm 0.8 \mathrm{MHz}$, and $T_{\mathrm{N}}^{\mathrm{ZF}}$ as $35.768 \pm 0.002 \mathrm{~K}$, although we need more accurate data in the vicinity of $T_{\mathrm{N}}$ to determine $\beta$ and $T_{\mathrm{N}}$ more precisely. ${ }^{55,56}$ The obtained $T_{\mathrm{N}}^{\mathrm{ZF}}$ is about $10 \mathrm{~K}$ lower than $T_{\mathrm{N}}(=45 \mathrm{~K})$ estimated by magnetization measurements with $H \geqslant 100$ Oe [see Fig. 2(a)]. Although the reason of the discrepancy between $T_{\mathrm{N}}$ determined by magnetization and $\mu^{+}$SR measurements is not clear at present, we will discuss this topic in Sec. III B. We wish, however, to point out that $T_{\mathrm{N}}^{\mathrm{ZF}}$ is consistent with $T_{\mathrm{N}}$ determined by neutron scattering $(36.5 \pm 0.5 \mathrm{~K})$ under zero applied field.

Although we fitted the ZF spectrum at each $T$ using Eq. (1), $A_{\mathrm{AF}, \mathrm{H}}, A_{\mathrm{AF}, \mathrm{L}}$, and $A_{\text {tail }}$ are found to be almost $T$ independent in the $T$ range between 1.8 and $31 \mathrm{~K}$ [see Fig. 4(c)]. In addition, $\lambda_{\text {tail }}$ is very small compared with $\lambda_{i}$ and roughly decreases with decreasing $T$ [see Fig. 4(b)]. Since $A_{\text {tail }} / A_{0} \sim 0.3$, the $A_{\text {tail }}$ signal is assigned to the " $1 / 3$ tail" signal, which is 
caused by the field component parallel to the initial muonspin polarization. Here, $A_{0} \sim 0.24$ was deduced from the measurements above $T_{\mathrm{N}}$ (see Sec. III B) and a reference silver sample. This also indicates the presence of a lost asymmetry ( $A_{\text {lost }}$ ) in the $\mathrm{ZF}$ spectrum below $T_{\mathrm{N}}$, which is simply estimated by $A_{\text {lost }}=A_{0}-A_{\mathrm{AF}, \mathrm{H}}-A_{\mathrm{AF}, \mathrm{L}}-A_{\text {tail }}$. We should note that a full asymmetry was observed above $T_{\mathrm{N}}$. Furthermore, a fast relaxing signal is unobservable in an early time domain of the $\mathrm{ZF}$ spectrum at $2 \mathrm{~K}$ [see Fig. 3(a)]. This implies the presence of a muonium state, i.e., a hydrogen-like " $\mu^{+} e^{-}$ atom," which sometimes exists in an insulator. According to the $T$ dependence of resistivity $(\rho)$ for $\mathrm{Li}_{2} \mathrm{MnO}_{3}$ above $\sim 475 \mathrm{~K},{ }^{57}$ $\rho$ increases with decreasing $T$ due to its insulating nature. Thus it is reasonable that a muonium state appears only at low $T$. In fact, the appearance of a muonium state only at low $T$ is also reported for $\mathrm{ZnO}$ below $30 \mathrm{~K},{ }^{58}$ and for $\mathrm{GaN}$ below $25 \mathrm{~K}^{59}$ Since the muonium Larmor frequency is faster by about 100 times than that of the muon, a fast relaxing signal is naturally too fast to be visible by conventional $\mu^{+} \mathrm{SR}$. The other possible explanation for $A_{\text {lost }}$ that some muons locate at the site with a very high internal field will be discussed in Appendix.

As $T$ increases from $2 \mathrm{~K}$, the relaxation rate of the AF signals is almost $T$ independent until $\sim 30 \mathrm{~K}$, and then increases with further increasing $T$ towards $T_{\mathrm{N}}$. This is a common behavior for the critical phenomenon at the AF transition. The initial phase $\left(\phi_{i}\right)$ for each oscillatory signal ranges below $\pm 20^{\circ}$ except in the vicinity below $T_{\mathrm{N}}$, where its determination is difficult, suggesting commensurate AF order to the lattice. This is because a "one channel offset" of the $\mu^{+}$SR spectrometer leads to a phase shift $\phi \sim 15^{\circ}$ for the oscillatory signal with $f=40 \mathrm{MHz}$. Therefore $\phi_{i}$ is assigned to be eventually zero. We will further discuss the AF spin structure for $\mathrm{Li}_{2} \mathrm{MnO}_{3}$ using the present $\mu^{+} \mathrm{SR}$ result in Sec. IV B.

\section{B. Magnetic behavior above $T_{\mathrm{N}}$}

In order to study the magnetic behavior above $T_{\mathrm{N}}$, we also measured the $\mu^{+}$SR spectrum until $70 \mathrm{~K}$ in a weak transverse field (wTF around 30 Oe) in a field cooling mode. Here, "weak" means that the applied field is significantly lower than any possible spontaneous internal fields $\left(H_{\text {int }}\right)$ in the ordered state. The wTF- $\mu^{+}$SR technique is sensitive to local magnetic order via the decrease in the amplitude (asymmetry) of the $\mu^{+}$spin precession signal and the enhanced $\mu^{+}$spin relaxation.

The wTF- $\mu^{+}$SR spectrum was fitted using a combination of a slowly relaxing precessing signal due to the applied wTF and Eq. (1):

$$
\begin{aligned}
A_{0} P_{\mathrm{TF}}(t)= & A_{\mathrm{TF}} \cos \left(2 \pi f_{\mathrm{TF}} t+\phi_{\mathrm{TF}}\right) \exp \left(-\frac{\sigma_{\mathrm{TF}}^{2} t^{2}}{2}\right) \\
& +A_{\mathrm{AF}, \mathrm{H}} \cos \left(2 \pi f_{\mathrm{H}} t+\phi_{\mathrm{H}}\right) \exp \left(-\lambda_{\mathrm{H}} t\right) \\
& +A_{\mathrm{AF}, \mathrm{L}} \cos \left(2 \pi f_{\mathrm{L}} t+\phi_{\mathrm{L}}\right) \exp \left(-\lambda_{\mathrm{L}} t\right) \\
& +A_{\text {tail }} \exp \left(-\lambda_{\text {tail }} t\right),
\end{aligned}
$$

where $P_{\mathrm{TF}}(t)$ is the muon spin polarization function under wTF, $f_{\mathrm{TF}}$ is the muon Larmor frequency corresponding to the applied wTF, $\phi_{\mathrm{TF}}$ is the initial phase of the precessing signal, $\sigma_{\mathrm{TF}}$ is the Gaussian relaxation rate, and $A_{\mathrm{TF}}$ is the asymmetry
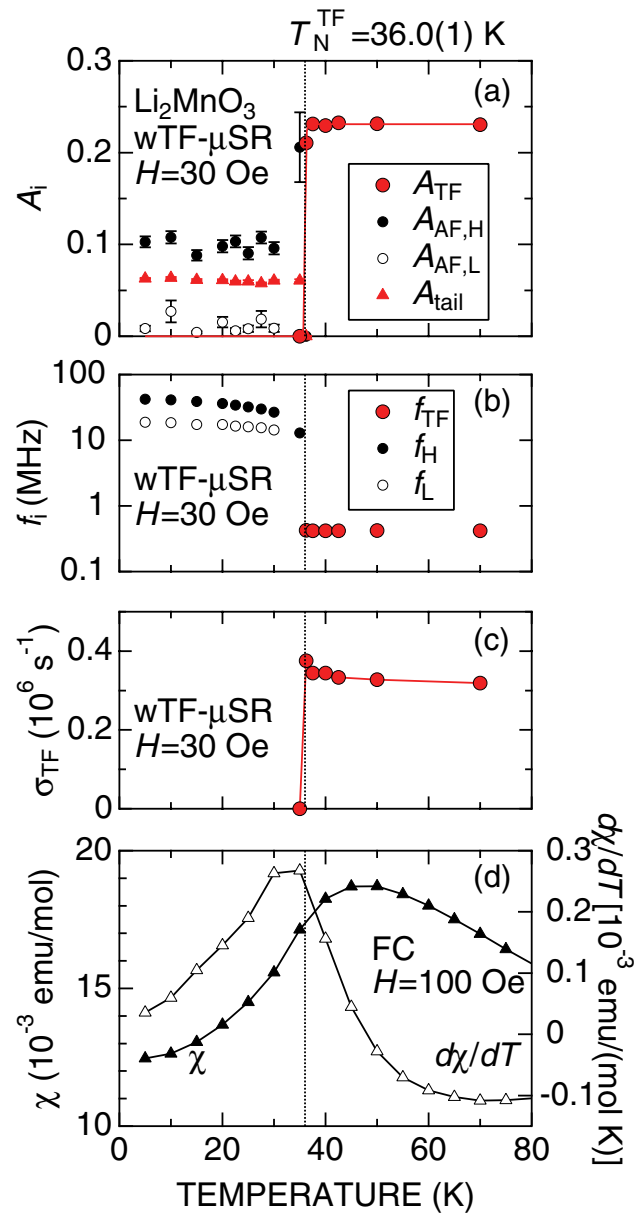

FIG. 5. (Color online) $T$ dependencies of (a) the asymmetries $\left(A_{i}\right)$, (b) the muon-spin precession frequencies $\left(f_{i}\right),(\mathrm{c})$ Gaussian relaxation rate $\left(\sigma_{\mathrm{TF}}\right)$ for the muon-spin precession signal due to $\mathrm{WTF}$, and (d) susceptibility $(\chi)$ and its slope $(d \chi / d T)$ for $\mathrm{Li}_{2} \mathrm{MnO}_{3}$. Each data in (a)-(c) was obtained by fitting the wTF-spectra using Eq. (2). In (b), since $\mathrm{wTF}=30 \mathrm{Oe}, f_{\mathrm{TF}} \sim 0.41 \mathrm{MHz}$. The $\chi$ data obtained with $H=100$ Oe is the same to that in Fig. 2.

of the wTF component of the $\mu^{+}$SR spectrum. Here, $A_{\mathrm{TF}}>0$ at $T>T_{\mathrm{N}}$, but $A_{\mathrm{TF}}=0$ at $T \leqslant T_{\mathrm{N}}$. On the contrary, $A_{\mathrm{AF}, \mathrm{H}}=$ $A_{\mathrm{AF}, \mathrm{L}}=A_{\text {tail }}=0$ at $T \geqslant T_{\mathrm{N}}$, as seen in Fig. 4 .

Figure 5 shows the $T$ dependencies of wTF- $\mu^{+}$SR parameters together with the $\chi(T)$ and $d \chi / d T(T)$ curves obtained with $H=100$ Oe in a field cooling mode. As $T$ decreases from $70 \mathrm{~K}, A_{\mathrm{TF}}$ is almost $T$ independent down to $37.5 \mathrm{~K}$, slightly decreases at $36.5 \mathrm{~K}$, and then suddenly drops to zero at $35 \mathrm{~K}$. At the same $T$, both $A_{\mathrm{AFh}}$ and $A_{\text {tail }}$ signals appear, while the $A_{\mathrm{AFl}}$ signal is observed at $T \leqslant 30 \mathrm{~K}$, indicating the formation of static AF order around $35 \mathrm{~K}$. Below $35 \mathrm{~K}$, the $T$ dependencies of $A_{i}$ and $f_{i}$ are reproducible to those of $A_{i}$ and $f_{i}$ extracted from the $\mathrm{ZF}-\mu^{+} \mathrm{SR}$ spectra, as expected. This is because the applied wTF is negligibly small compared with the static AF field [see Fig. 5(b)].

Here, $A_{\mathrm{TF}}(T) / A_{\mathrm{TF}}(70 \mathrm{~K})$ corresponds to the volume fraction of paramagnetic phases in a sample. Therefore $T_{\mathrm{N}}^{\mathrm{TF}}$ is estimated as $36.0(1) \mathrm{K}$, at which $A_{\mathrm{TF}}(T) / A_{\mathrm{TF}}(70 \mathrm{~K})=0.5$. The steplike change in the $A_{\mathrm{TF}}(T)$ curve at $T_{\mathrm{N}}^{\mathrm{TF}}$ and a small increase in $\sigma_{\mathrm{TF}}$ towards $T_{\mathrm{N}}^{\mathrm{TF}}$ [see Fig. 5(c)] indicate the absence of short-range order even above the vicinity of 

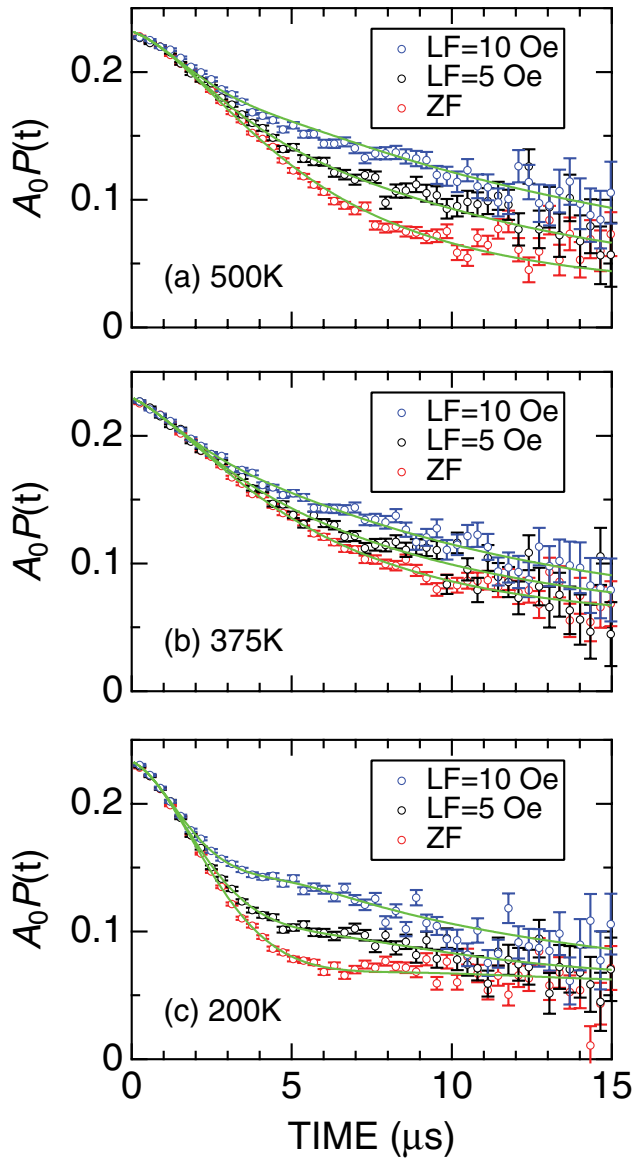

FIG. 6. (Color online) ZF- and LF- $\mu^{+} \mathrm{SR}$ spectra for $\mathrm{Li}_{2} \mathrm{MnO}_{3}$ obtained at (a) 500, (b) 375, and (c) $200 \mathrm{~K}$. The magnitude of LF were 5 and 10 Oe. Solid lines represent the fit result using Eq. (3). These spectra were obtained at ISIS/RAL.

$T_{\mathrm{N}}^{\mathrm{TF}}$. Note that $T_{\mathrm{N}}^{\mathrm{TF}}$ is comparable to $T_{\mathrm{N}}$ obtained from the $\mathrm{ZF}$ measurements $\left(T_{\mathrm{N}}^{\mathrm{ZF}}=35.768 \pm 0.002 \mathrm{~K}\right)$. On the other hand, the $\chi(T)$ curve shows a broad maximum around $45 \mathrm{~K}$ $\left(=T_{\mathrm{N}}^{\chi}\right)$ even with $H=100$ Oe. Since the $\chi(T)$ curve obtained with $H=10 \mathrm{kOe}$ is almost identical to that obtained with $H=100$ Oe (see Fig. 2), the discrepancy between $T_{\mathrm{N}}^{\mathrm{TF}}$ (or $T_{\mathrm{N}}^{\mathrm{ZF}}$ ) and $T_{\mathrm{N}}^{\chi}$ is an intrinsic feature for $\mathrm{Li}_{2} \mathrm{MnO}_{3}$. Interestingly, the $d \chi / d T(T)$ curve exhibits a maximum at $T_{\mathrm{N}}^{\mathrm{TF}}$, as in the case for a zigzag-chain compound, $\mathrm{CaCr}_{2} \mathrm{O}_{4}{ }^{60}$ This would suggest the effect of low dimensionality on $\chi$ in $\mathrm{Li}_{2} \mathrm{MnO}_{3}$.

\section{C. $\mathrm{Li}^{+}$diffusion at high temperature}

In order to know the temperature dependence of the $\mu^{+} \mathrm{SR}$ spectrum in the paramagnetic state, Fig. 6 shows the ZF and LF spectrum for $\mathrm{Li}_{2} \mathrm{MnO}_{3}$ obtained at 200, 375, and $500 \mathrm{~K}$. At each $T$, the ZF spectrum shows a damped Kubo-Toyabe behavior. This is characteristic for the case that the muon spins are depolarized by internal fields of both electronic and nuclear origin. ${ }^{48}$ Here, the former is caused by a fluctuating magnetic field due to $3 d$ electrons of $\mathrm{Mn}^{3+}$ ions $\left(H_{\mathrm{int}}^{\mathrm{E}}\right)$, while the latter is caused by nuclear magnetic fields due to ${ }^{6} \mathrm{Li},{ }^{7} \mathrm{Li},{ }^{55} \mathrm{Mn}$, and ${ }^{17} \mathrm{O}\left(H_{\text {int }}^{\mathrm{N}}\right)$. Since the natural abundance of ${ }^{6} \mathrm{Li},{ }^{7} \mathrm{Li},{ }^{55} \mathrm{Mn}$, and ${ }^{17} \mathrm{O}$ is $7.59 \%, 92.41 \%, 100 \%$, and $0.038 \%$, respectively, the effect of ${ }^{17} \mathrm{O}$ on $H_{\text {int }}^{\mathrm{N}}$ is negligibly small.
Furthermore, the applied LF ( $=10$ or 5 Oe) clearly reduces the relaxation rate, i.e., a decoupling behavior is observed at $200 \mathrm{~K}$. Although such a "decoupling" effect is very weak at $375 \mathrm{~K}$, indicating the increase in a field fluctuation rate of $H_{\mathrm{int}}^{\mathrm{N}}$ with $T$, the same LF decouples the relaxation rate again at $500 \mathrm{~K}$. This means that $H_{\mathrm{int}}^{\mathrm{N}}$ shows a static nature at $200 \mathrm{~K}$, but dynamic at $300 \mathrm{~K}$, and then becomes static again at $500 \mathrm{~K}$, as in the case for olivine-type $\mathrm{Li}_{M \mathrm{PO}_{4}}$ with $M=\mathrm{Fe}, \mathrm{Co}$, and $\mathrm{Ni}^{51,53}$

In order to know the change in $H_{\text {int }}^{\mathrm{E}}$ and $H_{\text {int }}^{\mathrm{N}}$ with $T$, the ZF and LF spectra were fitted simultaneously by a combination of an exponentially damped dynamic Gaussian Kubo-Toyabe function $\left[G^{\mathrm{DGKT}}\left(\Delta, v, t, H_{\mathrm{LF}}\right)\right]$ caused by a fluctuating $H_{\mathrm{int}}^{\mathrm{N}}$ due to ${ }^{6} \mathrm{Li},{ }^{7} \mathrm{Li}$, and ${ }^{55} \mathrm{Mn}$ nuclear moments, ${ }^{48}$ and an offset background (BG) signal from the fraction of muons stopped mainly in the sample cell, which is made of high-purity titanium:

$A_{0} P_{\mathrm{LF}}(t)=A_{\mathrm{KT}} \exp (-\lambda t) G^{\mathrm{DGKT}}\left(\Delta, v, t, H_{\mathrm{LF}}\right)+A_{\mathrm{BG}}$,

where $A_{0}$ is the initial $(t=0)$ asymmetry, $A_{\mathrm{KT}}$ and $A_{\mathrm{BG}}$ are the asymmetries associated with the three signals. $\lambda$ is the exponential relaxation rate, $\Delta$ is the static width of the local field distribution at the disordered sites, and $v$ is the field fluctuation rate. When $v=0$ and $H_{\mathrm{LF}}=0, G^{\mathrm{DGKT}}\left(t, \Delta, v, H_{\mathrm{LF}}\right)$ is the static Gaussian KT function $G_{z z}^{\mathrm{KT}}(t, \Delta)$ in ZF. The low- $T$ result suggests the presence of two different muon sites in the lattice. Although such situation is expected to be the same at $T$ above $T_{\mathrm{N}}, \Delta$ for the first site is predicted to be very similar to that for the second site (see Sec. IV A). Therefore the ZF and LF spectra at high $T$ are well fitted by Eq. (3).

At first, we fitted the ZF and LF spectra using a common $A_{\mathrm{BG}}$ in the whole $T$ range and common, i.e., $H_{\mathrm{LF}}$ independent, $\Delta$ and $v$ at each $T$ in Eq. (3). As a result, $\lambda$ was found to be rather small compared with $\Delta$ and $v$ and almost $T$ independent in the whole $T$ range. Therefore we finally fitted all the $\mathrm{ZF}$ and LF spectra using common $A_{\mathrm{KT}}, A_{\mathrm{BG}}$, and $\lambda$. Such a global fit provided that $A_{\mathrm{KT}}=0.1795 \pm 0.0003, A_{\mathrm{BG}}=0.0595 \pm$ 0.0003 , and $\lambda=(0.0563 \pm 0.0019) \times 10^{6} \mathrm{~s}^{-1}$. Here, the origin of $\lambda$ is a coupling between localized Mn moments and muon-spins, where the coupling constant $J_{\mathrm{KT}}$ is expected to be very small, since $\lambda$ is $T$ independent.

Figure 7 shows the $T$ dependencies of $\Delta, v$, and $\lambda$ obtained by the global fit. As $T$ increases from $175 \mathrm{~K}, \Delta$ is almost

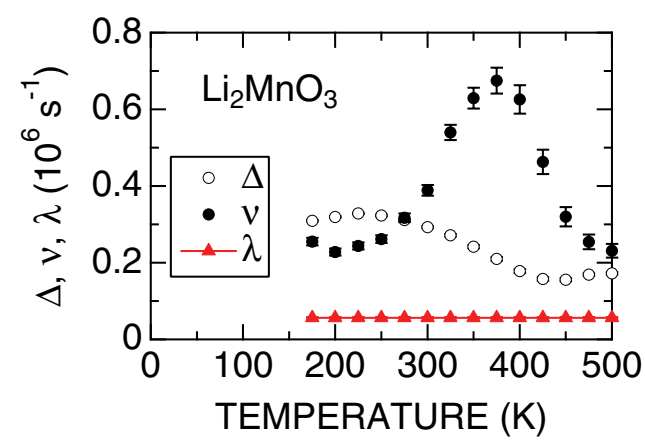

FIG. 7. (Color online) $T$ dependencies of $\Delta$ and $v$ for $\mathrm{Li}_{2} \mathrm{MnO}_{3}$. The common parameter $\lambda=(0.0563 \pm 0.0019) \times 10^{6} \mathrm{~s}^{-1}$ is also plotted for comparison. Each set of data was obtained by global fitting the ZF and LF spectra using Eq. (3). 


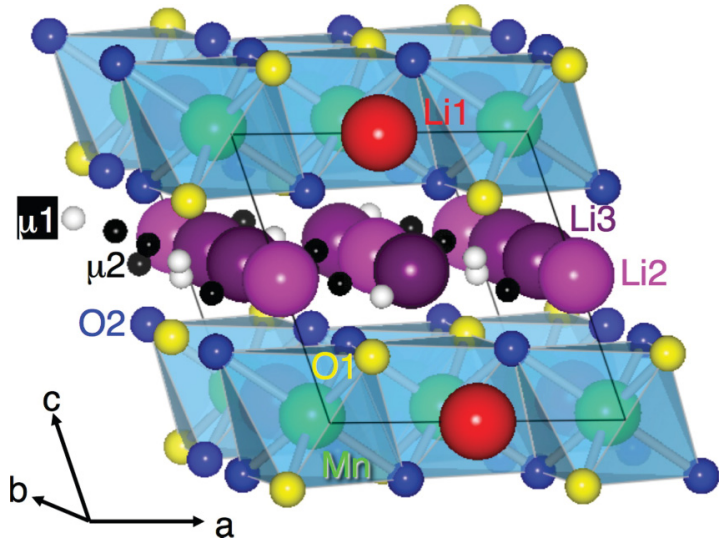

FIG. 8. (Color online) Possible muon sites $(\mu 1$ and $\mu 2)$ for $\mathrm{Li}_{2} \mathrm{MnO}_{3}$ predicted by electrostatic potential calculations. Also, since there are three crystallographically different $\mathrm{Li}$ sites and two crystallographically different $\mathrm{O}$ sites in the lattice, they are represented by different colors for clarity of display. Solid lines represent a crystallographic unit cell.

independent of $T$ until $\sim 250 \mathrm{~K}$, and decreases slightly with $T$, then finally levels off to a constant value $\left(\sim 0.16 \times 10^{-6} \mathrm{~s}^{-1}\right)$ above $\sim 425 \mathrm{~K}$. On the other hand, $v$ starts to increase above around $250 \mathrm{~K}$ with increasing the slope $(d v / d T)$, reaches a maximum at $375 \mathrm{~K}$ and then decreases with further increasing $T$. Then, $v$ seems to level off above $475 \mathrm{~K}$. Since the static behavior at high $T$ excludes the possibility of muon-diffusion, the $\mathrm{Li}^{+}$ions are found to diffuse above $\sim 300 \mathrm{~K}$ and their diffusion rate increases with $T$, as in the case for $\mathrm{Li} M \mathrm{PO}_{4}{ }^{51,53}$ When $v$ becomes rather large compared with $\Delta$, such diffusion is too fast to be visible by $\mu^{+} \mathrm{SR}$, resulting in the decrease in $v$ with further increasing $T$ above $375 \mathrm{~K}$, and finally, $v(\Delta)$ corresponds to the nuclear field fluctuation rate (nuclear field distribution width) mainly by immobile $\mathrm{Li}$ and ${ }^{55} \mathrm{Mn}$ above $\sim 450 \mathrm{~K}$. The diffusive behavior detected by $\mu^{+} \mathrm{SR}$ will be discussed in detail in Sec. IV C.

\section{DISCUSSION}

\section{A. Muon sites}

In the $\mathrm{Li}_{2} \mathrm{MnO}_{3}$ lattice, there are two different oxygen sites, i.e., the $\mathrm{O} 1$ and $\mathrm{O} 2$ sites, as seen in Fig. 8 and Table I. Therefore, assuming that each $\mu$ is bound to the nearest $\mathrm{O}^{2-}$ ion with a typical bond length for oxides, namely, $d_{\mu-\mathrm{O}}=1 \AA,{ }^{54}$ electrostatic potential $(E)$ calculations using a point charge model suggested two possible muon sites in the vicinity of the $\mathrm{O}^{2-}$ ions, i.e., the $\mu 1$ and $\mu 2$ site, in the $\mathrm{Li}_{2} \mathrm{MnO}_{3}$ lattice (see Table I and Fig. 8).
The calculations also indicate that the number density of the $\mu 2$ site is twice larger than that of the $\mu 1$ site. Furthermore, $E$ for the $\mu 2$ site is slightly lower than $E$ for the $\mu 1$ site. Therefore the muons located at the $\mu 2$ site are most likely to provide a major signal in the $\mu^{+}$SR spectrum, particularly at low $T$. However, in a paramagnetic state, $\Delta(0 \mathrm{~K})$ at the $\mu 2$ site $\left(=0.598 \times 10^{6} \mathrm{~s}^{-1}\right)$ is very close to that at the $\mu 1$ site $\left(=0.562 \times 10^{6} \mathrm{~s}^{-1}\right)$. This is consistent with the fact that, at $T$ above $T_{\mathrm{N}}$, the $\mathrm{ZF}$ and $\mathrm{LF}$ spectra from $\mathrm{Li}_{2} \mathrm{MnO}_{3}$ were well fitted by one signal [see Eq. (3)]. Note that the dipole field calculations provide $\Delta$ at $0 \mathrm{~K}$, while the lowest $T$, at which $\Delta$ and $v$ were measured, was $175 \mathrm{~K}$ (see Fig. 7). As a result, due to a thermally activated motion of ions and muons, the measured $\Delta$ is smaller by $40 \%$ than $\Delta(0 \mathrm{~K})$. Such discrepancy is also observed for $\mathrm{Li}_{x} \mathrm{CoO}_{2}{ }^{29}$ and $\mathrm{Li} M \mathrm{PO}_{4}{ }^{51-53}$ (see also Appendix).

\section{B. AF spin structure}

Here, we wish to discuss the AF spin structure, based on the present $\mu^{+} \mathrm{SR}$ result. Since there are two muon sites in the $\mathrm{Li}_{2} \mathrm{MnO}_{3}$ lattice, which provide two distinct internal magnetic fields for the common AF spin structure (see Figs. 3 and 4), we have multiple viewpoints to conjecture the $\mathrm{AF}$ spin structure for $\mathrm{Li}_{2} \mathrm{MnO}_{3}$. This provides complimentary information to that obtained by neutron scattering, as in the case for $\mathrm{BaCoO}_{3},{ }^{62}$ $\mathrm{Ba}_{2} \mathrm{CoO}_{4},{ }^{63} \mathrm{NaV}_{2} \mathrm{O}_{4},{ }^{64,65} R \mathrm{CoAsO},{ }^{66}$ and $\mathrm{K}_{2} \mathrm{Cr}_{8} \mathrm{O}_{16} \cdot{ }^{67}$

Past neutron work reported the presence of magnetic Bragg peaks below $T_{\mathrm{N}}$ and suggested that the AF unit cell is doubled along the $c$ axis, due to opposite moments in the adjacent $\left[\mathrm{Li}_{1 / 3} \mathrm{Mn}_{2 / 3}\right] \mathrm{O}_{2}$ layers along the $c$ axis. In addition, among the six possible AF models deduced from symmetry analysis, either the $F_{z}$-type or $C_{z}$-type $\mathrm{AF}$ order was proposed as the candidate for the $\mathrm{AF}$ ground state of $\mathrm{Li}_{2} \mathrm{MnO}_{3}$. Here, for the $F_{z}$-type order, the Mn moments align parallel to the $c$ axis in the $\left[\mathrm{Li}_{1 / 3} \mathrm{Mn}_{2 / 3}\right] \mathrm{O}_{2}$ layer but antiparallel between the adjacent $\left[\mathrm{Li}_{1 / 3} \mathrm{Mn}_{2 / 3}\right] \mathrm{O}_{2}$ layers. On the contrary, for the $C_{z}$-type order, each Mn moment aligns parallel or antiparallel to the $c$ axis, and an FM chain along the $a$ axis aligns antiferromagnetically along both the $b$ and $c$ axis.

Table II summarizes the internal fields at the two muon sites $\left(H_{\mu i}\right.$ and $\left.f_{\mu i}\right)$ using the six AF models estimated by dipole field calculations. Since the ordered Mn moment $\left(\mu_{\mathrm{Mn}}^{\text {ord }}\right)$ should be less than $3 \mu_{\mathrm{B}}$ for $\mathrm{Mn}^{4+}$ with $S=3 / 2$, all the $F$ type AF models are thought to be not suitable for $\mathrm{Li}_{2} \mathrm{MnO}_{3}$. Moreover, the ratio between the two internal fields for each $F$-type disagrees with the experimental result, supporting the above conclusion. On the other hand, among the three $C$-type $\mathrm{AF}$ models, either $C_{x}$ or $C_{y}$ is a candidate for the $\mathrm{AF}$ order in

TABLE I. Possible muon sites $\left(\mu_{n}\right)$, which locate $1 \AA$ away from $\mathrm{O}_{n}$, the position of the nearest $\mathrm{O}^{2-}$, the position of $\mu_{n}$, the distance to the nearest $\mathrm{Mn}$ ions $\left(d_{\mu \text {-Mn }}\right)$ electrostatic potential $(E)$ at $\mu_{n}$, the number of the site per crystallographic unit cell, and the nuclear dipole field distribution width at $0 \mathrm{~K}(\Delta)$ for $\mathrm{Li}_{2} \mathrm{MnO}_{3}$ determined by electrostatic potential calculations and dipole field calculations. The calculations were performed by a computer program DIPELEC,${ }^{61}$ which uses a point charge model.

\begin{tabular}{lcccccc}
\hline \hline Site & Nearest O site $(x, y, z)$ & $(x, y, z)$ & $d_{\mu-\mathrm{Mn}}(\AA)$ & $E(\mathrm{eV})$ & Number/unit cell & $\Delta\left(10^{6}\right) \mathrm{s}^{-1}$ \\
\hline$\mu 1$ & O1 $(0.2189,0.0000,0.2273)$ & $(0.3213,0.0000,0.4355)$ & 2.6441 & -10.558 & 4 \\
$\mu 2$ & O2 $(0.2540,0.3211,0.2233)$ & $(0.2974,0.3452,0.4278)$ & 2.6518 & -10.624 & 8 & 0.598 \\
\hline \hline
\end{tabular}


TABLE II. The internal magnetic field at the two muon sites for the six AF models proposed by neutron. The AF model $C_{n}$ and $F_{n}$ with $n=x, y, z$, the internal magnetic field $(H)$ at the $\mu 1$ and $\mu 2$ site, the maximum value of the muon-spin precession frequency $\left(f_{\mu i}^{\max }\right)$ at the $\mu 1$ and $\mu 2$ site, the ratio between the two $f_{\mu i}^{\max } \mathrm{s}$, and the ordered Mn moment $\left(\mu_{\mathrm{Mn}}^{\mathrm{ord}}\right)$. Note that the AF unit cell is twice as large along the $c$ axis. The $F_{n}$ order corresponds to the $A$-type $\mathrm{AF}$ order along the $c$ axis, namely, the $\mathrm{Mn}$ moments align ferromagnetically in the $\left[\mathrm{Li}_{1 / 3} \mathrm{Mn}_{2 / 3}\right] \mathrm{O}_{2} l_{\text {layer }}$ but antiferromagnetically between the adjacent $\left[\mathrm{Li}_{1 / 3} \mathrm{Mn}_{2 / 3}\right] \mathrm{O}_{2}$ layers. For the $F_{x}\left(F_{y}, F_{z}\right)$ order, the Mn moments align parallel or antiparallel to the $a(b, c)$ axis. On the other hand, for the $C_{x}\left(C_{y}, C_{z}\right)$ order, each Mn moment aligns parallel or antiparallel to the $a(b, c)$ axis, and an FM chain along the $a$ axis aligns antiferromagnetically along both the $b$ and $c$ axis. $f_{\mu i}^{\max }$ is $f_{\mu i}$ when $\mu_{\mathrm{Mn}}^{\text {ord }}$ is the theoretical value of Mn ${ }^{4+}$ with $S=3 / 2\left(3 \mu_{\mathrm{B}}\right)$.

\begin{tabular}{lccccc}
\hline \hline AF order & $H_{\mu 1}\left(\mathrm{Oe} / \mu_{\mathrm{B}}\right)$ & $H_{\mu 2}\left(\mathrm{Oe} / \mu_{\mathrm{B}}\right)$ & $f_{\mu 1}^{\max }(\mathrm{MHz})$ & $f_{\mu 2}^{\max }(\mathrm{MHz})$ & $f_{\mu 1} / f_{\mu 2}$ \\
\hline$F_{x}$ & 645 & 598 & 26.2 & 24.3 & 1.078 \\
$F_{y}$ & 207 & 601 & 8.4 & 24.4 & 0.345 \\
$F_{z}$ & 548 & 873 & 22.3 & 35.5 & 0.628 \\
$C_{x}$ & 566 & 1187 & 23.0 & 48.3 & 3.18 \\
$C_{y}$ & 1040 & 495 & 42.3 & 20.1 & 0.476 \\
$C_{z}$ & 873 & 1110 & 35.5 & 45.1 & 2.104 \\
experiment & & $19.09 \pm 0.05$ & $42.14 \pm 0.06$ & 0.787 & 0.453 \\
\hline \hline
\end{tabular}

$\mathrm{Li}_{2} \mathrm{MnO}_{3}$. Here, we should remind that the muons at the $\mu 2$ site provide a major signal, leading to $H_{\mu 2}>H_{\mu 1}$. Therefore the most probable AF order for $\mathrm{Li}_{2} \mathrm{MnO}_{3}$ is determined as the $C_{x}$-type order, as shown in Fig. 9. The $C_{x}$-type order is reasonably formed when the interaction between the nearest neighboring $\mathrm{Mn}$ ions is $\mathrm{AF}$ and the interaction via the Mn-OLi-O-Mn path is AF both along the $c$ axis and in the $a b$ plane. Indeed, $\mu_{\mathrm{Mn}}^{\text {ord }}$ is estimated as $2.62 \mu_{\mathrm{B}}$ at $1.8 \mathrm{~K}$, which is in good agreement with the neutron result $\left(2.7 \pm 0.3 \mu_{\mathrm{B}}\right)$ at $4 \mathrm{~K}$.

According to the simulation of the neutron diffraction patterns with the wavelength $\lambda_{\mathrm{n}}=2.522 \AA$, the $F_{z}$-type order yields a magnetic diffraction peak at $2 \theta=15.1^{\circ}$ with the intensity of $75 \%$ of that at $2 \theta=34.1^{\circ}$. The peak at $2 \theta=15.1^{\circ}$

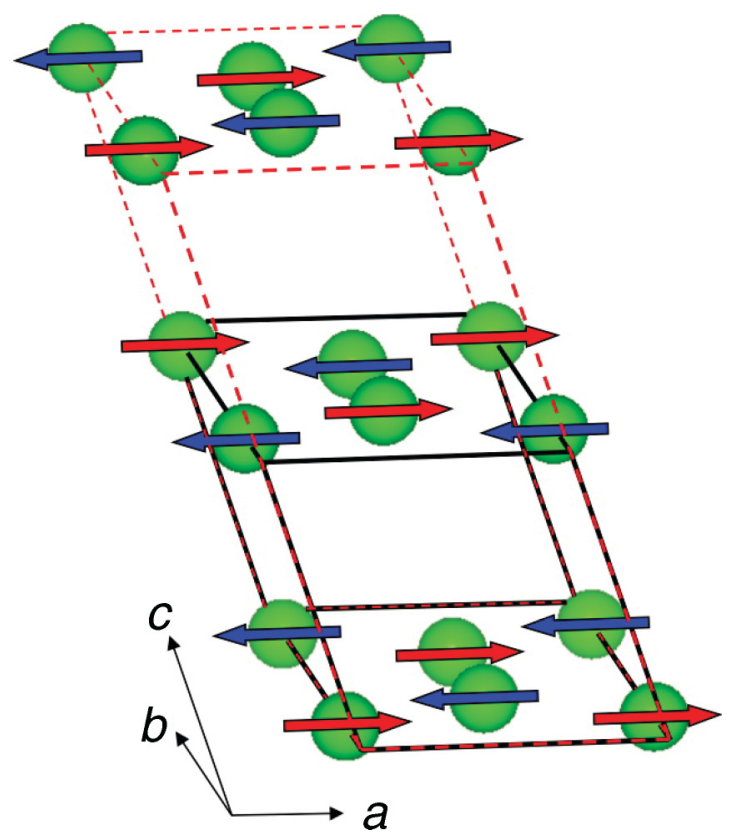

FIG. 9. (Color online) The $C_{x}$-type AF spin structure for $\mathrm{Li}_{2} \mathrm{MnO}_{3}$ proposed by the present $\mu^{+} \mathrm{SR}$ investigation. Red and blue arrows correspond to the Mn moment. Note that only Mn ions are shown for clarity of display. Solid lines represent a crystallographic unit cell, while broken lines represent a magnetic unit cell. is the most intense magnetic diffraction peak for both the $F_{x}$ and $F_{y}$-type order. On the other hand, such diffraction peak no longer exists for the $C$-type order. Moreover, either $C_{x}$ or $C_{z}$-type order provides an acceptable result for explaining the reported diffraction pattern. Therefore the $F$-type order is highly unlikely to fit the experimental data, but either the $C_{x}$ or $C_{z}$-type order is more likely. Consequently, the $C_{x}$-type $\mathrm{AF}$ order is very reasonable both for the present $\mu^{+}$SR result and past neutron data.

Furthermore, we consider the effect of the FM arrangement in the $F$-type order on $H_{\mu}$, namely, the hyperfine field $\left(H_{\mathrm{hf}}\right)$ due to the local spin density at the muon site might significantly contribute to $H_{\mu}$, as in the case of ferromagnetic $R \mathrm{CoAsO}$ and $\mathrm{K}_{2} \mathrm{Cr}_{8} \mathrm{O}_{16} \cdot{ }^{66,67}$ For the compound with an $\alpha-\mathrm{NaFeO}_{2}$-type structure, $F$-type AF order is known to be formed in $\mathrm{NaNiO}_{2}$ below $T_{\mathrm{N}} \sim 23 \mathrm{~K} .{ }^{24,68}$ However, the observed $H_{\mu}(\sim 60 \mathrm{MHz}$ at $2 \mathrm{~K}$ ) was in good agreement with the prediction from dipole field calculations. ${ }^{40}$ This suggests that $H_{\mathrm{hf}}$ is not so large compared with that for ferromagnetic materials. Hence it is reasonable that the $F$-type order is excluded for $\mathrm{Li}_{2} \mathrm{MnO}_{3}$, based on the comparison between the measured $H_{\mu}$ and the prediction from dipole field calculations.

\section{Li diffusive behavior}

We attempt to evaluate a self-diffusion coefficient of $\mathrm{Li}^{+}$ ions $\left(D_{\mathrm{Li}}\right)$ using the present $\mu^{+} \mathrm{SR}$ result. At first, we need to determine the diffusion pathway(s) of $\mathrm{Li}^{+}$. Here, we should note that there are three different $\mathrm{Li}$ sites in the lattice (see Fig. 8). Also, since the regular Li1 site is fully occupied by $\mathrm{Li}$, we naturally consider only the jump to interstitial sites. The electrostatic potential calculations suggest six possible interstitial sites for the $\mathrm{Li} 2$ and $\mathrm{Li} 3$ sites in the $a b$ plane, whereas two possible interstitial sites for the Li1 and Li3 site in the $b c$ plane (see Fig. 10).

Then, we focus on the decrease in $\Delta$ due to Li diffusion. That is, when $\mathrm{Li}^{+}$ions diffuse too fast, i.e., $v \gg \Delta$, such diffusion is invisible by $\mu^{+} \mathrm{SR}$. As a result, $\Delta$ gradually decreases with $T$ above $250 \mathrm{~K}$, and finally, $\Delta$ approaches to the $\Delta\left(\sim 0.18 \times 10^{6} \mathrm{~s}^{-1}\right)$ caused by immobile Li and ${ }^{55} \mathrm{Mn}$. In 


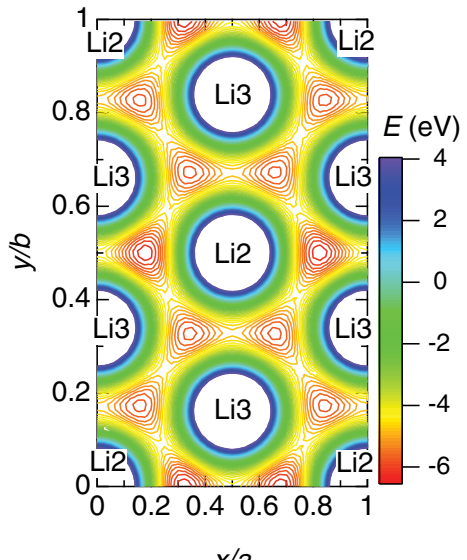

$x / a$

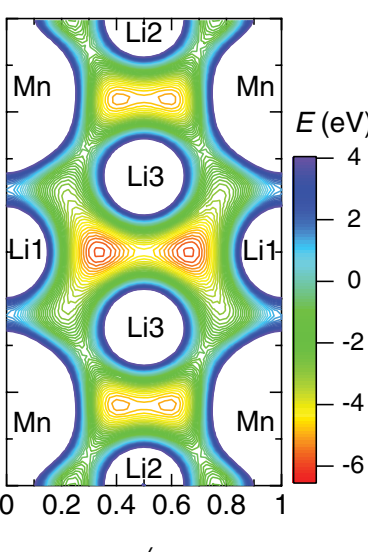

$z / C$

FIG. 10. (Color online) Electrostatic potential distribution in (a) the $(x, y, 0.5)$ plane and (b) the $(0, y, z)$ plane for $\mathrm{Li}_{2} \mathrm{MnO}_{3}$. The lattice constants are $a=4.937 \AA, b=8.532 \AA, c=5.030 \AA$, and $\beta=109.46^{\circ}$.

other words, we could deduce the mobile $\mathrm{Li}^{+}$from the change in $\Delta$. Thus the magnitude of $\Delta$ was estimated for the $\mathrm{Li}_{x} \mathrm{MnO}_{3}$ phase without $\mathrm{Li} 1, \mathrm{Li} 2$, and/or $\mathrm{Li} 3$ by dipole field calculations (see Table III). Comparing with the experimental result at 200 and $500 \mathrm{~K}$, it is clearly excluded that the $\mathrm{Li}^{+}$ions at the $\mathrm{Li} 2$ and $\mathrm{Li} 3$ sites, i.e., all the $\mathrm{Li}^{+}$ions in the $\mathrm{Li}$ plane, become mobile at high $T$, because $\Delta_{\mu i}(-\mathrm{Li} 2,-\mathrm{Li} 3)$ are too small for explaining the experimental result. Also, it is most unlikely that only the $\mathrm{Li}^{+}$ions at the $\mathrm{Li} 2$ site are mobile at high $T$.

Therefore the most reasonable scenario is that the $\mathrm{Li}^{+}$ions at the $\mathrm{Li} 3$ and $\mathrm{Li} 1$ sites are mobile at high $T$, but the $\mathrm{Li}^{+}$ ions at the Li2 site are still immobile even at $500 \mathrm{~K}$. In other words, the $\mathrm{Li}^{+}$ions are diffusing not in the $\mathrm{Li}$ plane, i.e., in the $a b$ plane, but along the $c$ axis through the $\mathrm{Li}^{+}$ions in the $\left[\mathrm{Li}_{1 / 3} \mathrm{Mn}_{2 / 3}\right]$ plane (Li1 site). This is consistent with the prediction from first-principles calculations ${ }^{69}$ in which $\mathrm{Li}_{x} \mathrm{MnO}_{3}$ with $\mathrm{Li}$ vacancies in both the $\mathrm{Li}$ and $\left[\mathrm{Li}_{1 / 3} \mathrm{Mn}_{2 / 3}\right]$ planes is more stable than that with $\mathrm{Li}$ vacancies only in the $\mathrm{Li}$ plane. Thus we will consider the two diffusion pathways in the $b c$ plane [see Fig. 10(b)]; namely, one is the pathway from the Li1 site to the interstitial site at $(0.0019,0.5000,0.3374)$ along the $c$ axis, and the other is the pathway from the Li3 site to the same interstitial site.

Assuming that $v$ corresponds to the jump rate of the $\mathrm{Li}^{+}$ ions between the neighboring sites, $D_{\mathrm{Li}}$ is given by ${ }^{70}$

$$
D_{\mathrm{Li}}=\sum_{i=1}^{n} \frac{1}{N_{i}} Z_{v, i} s_{i}^{2} \nu,
$$

where $N_{i}$ is the number of $\mathrm{Li}$ sites in the $i$ th path, $Z_{v, i}$ is the vacancy fraction, and $s_{i}$ is the jump distance. Based on the above consideration, $n=2, N_{1}=2$, and $Z_{1}=1$ and $N_{2}=2$, and $Z_{2}=1$. From Fig. $10, s_{1}=1.697 \AA$ and $s_{2}=1.596 \AA$. In order to extract $v$ due to $\mathrm{Li}$ diffusion, we fitted the $\nu$-versus- $1 / T$ curve by a combination of a thermal activation process due to $\mathrm{Li}$ diffusion and a $T$-independent offset signal caused by the fluctuation of $\mathrm{Mn}$ moments [see Fig. 11(a)]. That is, $v=v_{0} \exp \left(-E_{a} / k_{\mathrm{B}} T\right)+v_{\mathrm{Mn}}$, where $E_{\mathrm{a}}$ is the activation energy and $k_{\mathrm{B}}$ is the Boltzmann's constant. Such fit provides that $v_{0}=(75.577 \pm 0.001) \times 10^{6} \mathrm{~s}^{-1}, E_{a}=$ $0.156 \pm 0.003 \mathrm{eV}$, and $v_{\mathrm{Mn}}=(0.22 \pm 0.05) \times 10^{6} \mathrm{~s}^{-1}$. Using $\left(v-v_{\mathrm{Mn}}\right)$ instead of $v$ in Eq. (4), we have obtained the $T$ dependence of $D_{\mathrm{Li}}$ for $\mathrm{Li}_{2} \mathrm{MnO}_{3}$ [see Fig. 11(b)]. However, there are, to our knowledge, no available data on $D_{\mathrm{Li}}$ from neither other experiments, nor calculations.

Concerning $E_{a}$, the value obtained by $\mu^{+}$SR is about $1 / 3$ of $E_{a}(0.45 \mathrm{eV})$ estimated from ac impedance measurements for a powder sample. ${ }^{46}$ The discrepancy between $E_{a}$ obtained by $\mu^{+} \mathrm{SR}$ and ac impedance is due to the fact that $\mu^{+} \mathrm{SR}$ is specially sensitive to a short-range jump of $\mathrm{Li}^{+}$ions, while ac impedance senses the long-range Li diffusion. In other words, since $\mu^{+} \mathrm{SR}$ is a local probe, a powder sample is approximately equivalent to a single-crystal sample for muons. A very similar discrepancy between $E_{a}$ obtained by NMR and ac impedance is also reported for several materials. ${ }^{71}$

Finally, we wish to point out the diffusive nature of the Li-overstiochiometric $\mathrm{Li} M \mathrm{O}_{2}$ compounds, such as, $\mathrm{Li}_{1+\delta} \mathrm{Co}_{1-\delta} \mathrm{O}_{2-\delta}{ }^{72}$ and $\mathrm{Li}_{1+\delta}\left(\mathrm{Co}_{0.333} \mathrm{Ni}_{0.333} \mathrm{Mn}_{0.333}\right)_{1-\delta} \mathrm{O}_{2}{ }^{23}$ For these compounds, the excess $\mathrm{Li}^{+}$ions naturally locate at the $M$ site in the $M \mathrm{O}_{2}$ layers. Based on the present result, such $\mathrm{Li}^{+}$ions are likely to make an additional pathway of $\mathrm{Li}$ diffusion along the $c$ axis. In order to know the effect of $\delta$ on

TABLE III. The predicted variation of the field distribution width at $0 \mathrm{~K}(\Delta)$ with the vacant $\mathrm{Li}$ sites. Here, we assume that, when the $\mathrm{Li}^{+}$ ions diffuse too fast to be visible by $\mu^{+} \mathrm{SR}, \Delta$ decreases as if such Li ions are absent in the lattice. The experimental results obtained at 200 and $500 \mathrm{~K}$ are also listed for comparison. Here, since the muons at the $\mu 2$ site are expected to contribute to the major signal, $\Delta_{\mu 2}$ and $\Delta_{\mu 2} / \Delta_{\mu 2}^{\text {none }}$ should be compared with the experimental result.

\begin{tabular}{lccc}
\hline \hline Vacant Li & $\Delta_{\mu 1}\left(10^{6} \mathrm{~s}^{-1}\right)$ & $\Delta_{\mu 2}\left(10^{6} \mathrm{~s}^{-1}\right)$ & $\Delta_{\mu 1} / \Delta_{\mu 1}^{\text {none }}$ \\
\hline none & 0.598 & 0.562 & 1 \\
Li1 & 0.587 & 0.552 & 0.981 \\
Li2 & 0.526 & 0.449 & 0.879 \\
Li3 & 0.336 & 0.383 & 0.562 \\
Li2 and Li3 & 0.178 & 0.180 & 0.298 \\
Li3 and Li1 & 0.315 & 0.369 & 0.527 \\
Li1 and Li2 & 0.513 & 0.437 & 0.858 \\
Li1, L2, and Li3 & 0.135 & 0.148 & 0.226 \\
experiment & $\Delta(200 \mathrm{~K})\left(10^{6} \mathrm{~s}^{-1}\right)$ & $\Delta(500 \mathrm{~K})\left(10^{6} \mathrm{~s}^{-1}\right)$ & 0.682 \\
& $0.3411 \pm 0.0013$ & $0.1725 \pm 0.0034$ & 0.321 \\
& & & 0.056 \\
\hline \hline
\end{tabular}



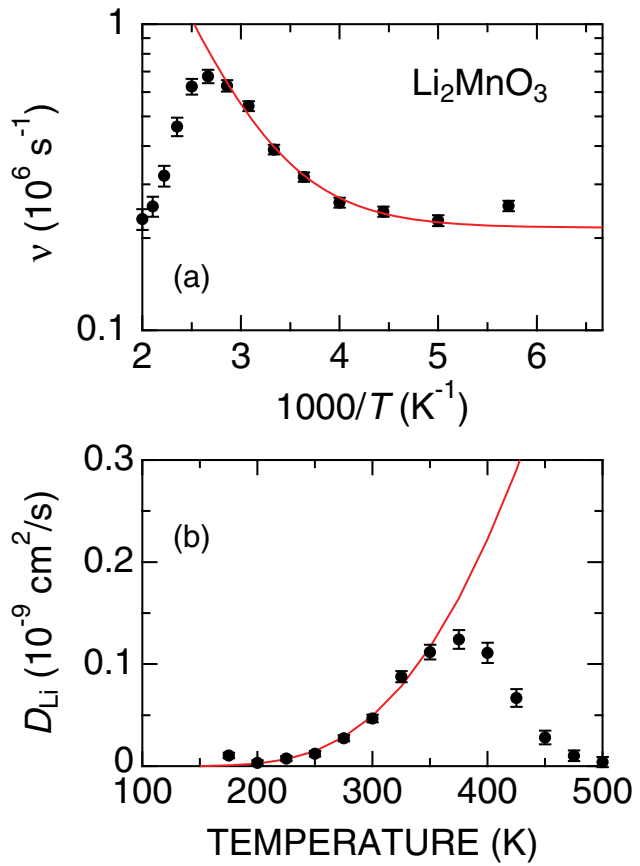

FIG. 11. (Color online) (a) The relationship between $v$ and $1 / T$ and (b) the $T$ dependence of $D_{\mathrm{Li}}$ for $\mathrm{Li}_{2} \mathrm{MnO}_{3}$. In (a), a solid line shows a combination of a thermal activation process and a $T$ independent offset discussed in the text. In (b), a solid line shows the predicted $D_{\mathrm{Li}}(T)$ curve from the thermal activation process.

the diffusive nature, it would be very interesting to measure $D_{\mathrm{Li}}$ as a function of $\delta$.

\section{SUMMARY}

We have investigated the low- $T$ magnetic nature and high- $T$ diffusive behavior of $\mathrm{Li}_{2} \mathrm{MnO}_{3}$ using a unique local magnetic probe, i.e., the muon-spin rotation and relaxation $\left(\mu^{+} \mathrm{SR}\right)$ technique. Detailed analysis plus dipole field calculations suggested the formation of the $C_{x}$-type AF order below $T_{\mathrm{N}}=$ $35.768 \pm 0.002 \mathrm{~K}$, contrary to the $F_{z}$-type order proposed by past neutron measurements. From the temperature dependence of a nuclear field relaxation in a paramagnetic state, $\mathrm{Li}^{+}$ions were found to start diffusing above $200 \mathrm{~K}$. Based on the temperature dependence of the field distribution width, the diffusion pathway was assigned along the $c$ axis. Using this diffusion pathway and the field fluctuation rate, a self-diffusion coefficient of $\mathrm{Li}^{+}$ions was estimated as $(4.7 \pm 0.4) \times 10^{-11}$ $\mathrm{cm}^{2} / \mathrm{s}$ at $300 \mathrm{~K}$.

\section{ACKNOWLEDGMENTS}

We thank the staff of PSI and ISIS for help with the $\mu^{+}$SR experiments. D.A. acknowledges financial support from the Romanian UEFISCDI Project No.PN-II-ID-PCE-2011-30583 (85/2011). This work was also supported by the "UltraSlow Muon Microscope" (No. 23108003) Grant-in Aid for Scientific Research on Innovative Areas from the Ministry of
Education, Culture, Sports, Science and Technology (MEXT) of Japan. All images involving crystal structure were made with VESTA. ${ }^{73}$

\section{APPENDIX: EFFECT OF $\mu$-O DISTANCE ON CALCULATED RESULTS}

Here, we wish to show the reliability of the assumption that each $\mu$ is bound to the nearest $\mathrm{O}^{2-}$ ion with the distance, $d_{\mu-\mathrm{O}}=1 \AA$. Figure 12 shows the $d_{\mu-\mathrm{O}}$ dependencies of $\Delta_{\mu i}$, $E_{\mu i}, f_{\mu i}$ for the $F_{n}$ order, and $f_{\mu i}$ for the $C_{n}$ order. As $d_{\mu-\mathrm{O}}$ increases, the distance between $\mu^{+}$and the nearest $\mathrm{Li}$ decreases, while that between $\mu^{+}$and the nearest Mn increases. As a result, the change in $\Delta_{\mu i}$ is about $10 \%$, even when $d_{\mu-\mathrm{O}}$ varies from 0.8 to $1.2 \AA$. Also, $E_{\mu 1}$ is very close to $E_{\mu 2}$ in the whole calculated range, while $E_{\mu 1}>E_{\mu 2}$ when $d_{\mu-\mathrm{O}} \leqslant 1.0 \AA$, and $E_{\mu 1}<E_{\mu 2}$ when $d_{\mu-\mathrm{O}}>1.0 \AA$.

For the $F_{n}$ order, there is no combination of $f_{\mu 1}$ and $f_{\mu 2}$ to satisfy the two precession frequencies, $f_{\mathrm{H}}$ and $f_{\mathrm{L}}$, in the $d_{\mu-\mathrm{O}}$ range between 0.8 and $1.2 \AA$. On the other hand, for the $C_{n}$ order, both the $C_{x}$ and $C_{y}$ orders agree with the experimental result. Since $f_{\mu i}$ is calculated for $\mu_{\mathrm{Mn}}^{\mathrm{ord}}=3 \mu_{\mathrm{B}}$, it is also found
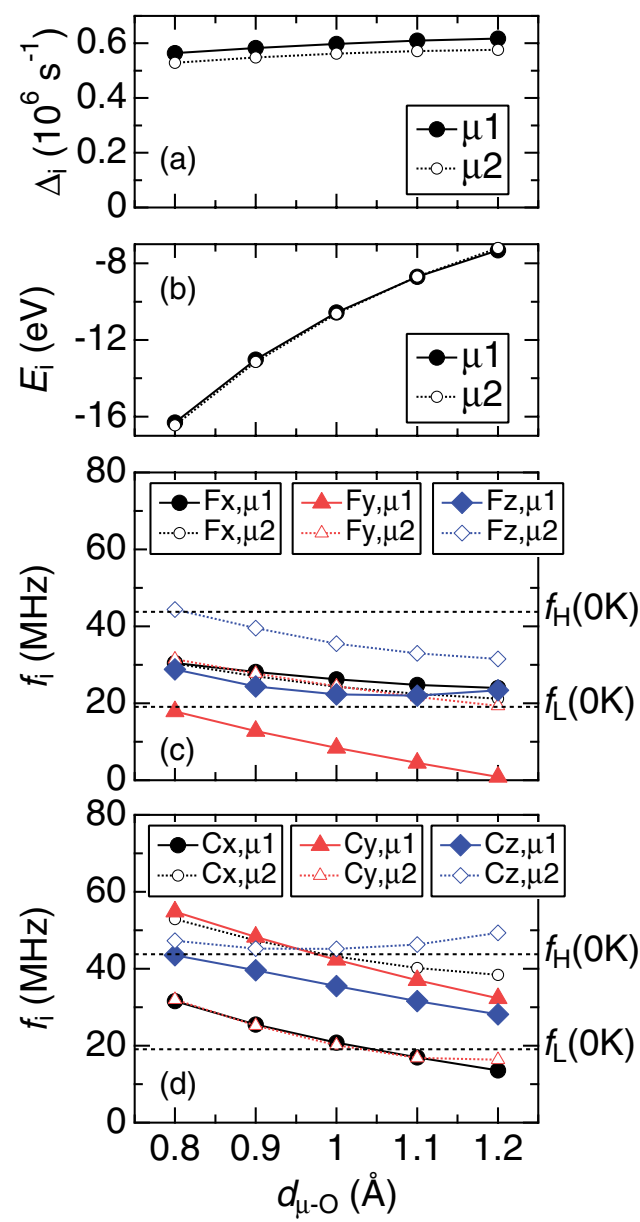

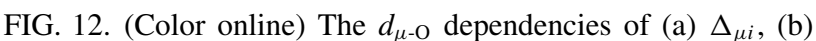
$E_{\mu i}$, (c) $f_{\mu i}$ for the $F_{n}$ order, and (d) $f_{\mu i}$ for the $C_{n}$ order. In (c) and (d), $\mu_{\mathrm{Mn}}^{\text {ord }}=3 \mu_{\mathrm{B}}$. 
that $d_{\mu-\mathrm{O}} \leqslant 1 \AA$. Considering $E_{\mu i}$ and the number of the $\mu 1$ and $\mu 2$ sites in the unit cell, the $C_{x}$ order is more likely for the AF spin structure than the $C_{y}$ order.

Figure 12 also indicates that the highest internal field at the muon site is $H_{\mu}=55 \mathrm{MHz}(=4.06 \mathrm{kOe})$ for the $C_{y}$ order with $d_{\mu-\mathrm{O}}=0.8 \AA$ or $H_{\mu}=53 \mathrm{MHz}(=3.91 \mathrm{kOe})$ for the $C_{x}$ order with $d_{\mu-\mathrm{O}}=0.8 \AA$. This means that all the signals from the muons in the $\mathrm{Li}_{2} \mathrm{MnO}_{3}$ lattice of the $\mathrm{AF}$-ordered phase range in the time window of $\mu^{+} \mathrm{SR}$. Therefore $A_{\text {lost }}$ below $T_{\mathrm{N}}$ is most unlikely to be caused by the muons located at the site with very high $H_{\mu}$, but highly likely to be caused by the muonium formation. *e0589@mosk.tytlabs.co.jp

${ }^{1}$ P. W. Anderson, Mat. Res. Bull. 8, 153 (1973).

${ }^{2}$ K. Hirakawa, H. Kadowaki, and K. Ubukoshi, J. Phys. Soc. Jpn. 54, 3526 (1985)

${ }^{3}$ H. R. Krishnamurthy, C. Jayaprakash, S. Sarker, and W. Wenzel, Phys. Rev. Lett. 64, 950 (1990).

${ }^{4}$ K. Mizushima, P. C. Jones, P. J. Wiseman, and J. B. Goodenough, Mat. Res. Bull. 15, 783 (1980).

${ }^{5}$ M. G. S. R. Thomas, W. I. F. David, and J. B. Goodenough, Mat. Res. Bull. 10, 1137 (1985).

${ }^{6}$ M. S. Whittingham, Chem. Rev. 104, 4271 (2004).

${ }^{7}$ V. Pralong, Prog. Solid State Chem. 37, 262 (2009).

${ }^{8}$ P. Strobel and B. Lambert-Andron, J. Solid State Chem. 75, 90 (1988).

${ }^{9}$ V. M. Jansen and R. Hoppe, Z. Anorg. Allg. Chem. 397, 279 (1973).

${ }^{10}$ V. G. Meyer and R. Hoppe, Z. Anorg. Allg. Chem. 424, 257 (1976).

${ }^{11}$ M. H. Rossouw and M. M. Thackeray, Mat. Res. Bull. 26, 467 (1991).

${ }^{12}$ Y. Shao-Horn, Y. Ein-EIi, A. D. Robertson, W. F. AveriII, S. A. Hackney, and J. W. F. Howard, J. Electrochem. Soc. 145, 16 (1998).

${ }^{13}$ P. Kalyani, S. Chitra, T. Mohan, and S. Gopukumar, J. Power Sources 80, 103 (1999).

${ }^{14}$ C. Johnson, S. Korte, J. Vaughey, M. M. Thackeray, T. Bofinger, Y. Shao-Horn, and S. Hackney, J. Power Sources 81-82, 491 (1999).

${ }^{15}$ S.-H. Park, Y. Sato, J.-K. Kim, and Y.-S. Lee, Mater. Chem. Phys. 102, 225 (2007).

${ }^{16}$ C. H. Lei, J. G. Wen, M. Sardela, J. Bareno, I. Petrov, S.-H. Kang, and D. P. Abraham, J. Mater. Sci. 44, 5579 (2009).

${ }^{17}$ D. Y. W. Yu, K. Yanagida, Y. Kato, and H. Nakamura, J. Electrochem. Soc. 156, A417 (2009).

${ }^{18}$ A. Boulineau, L. Croguennec, C. Delmas, and F. Weill, Solid State Ionics 180, 1652 (2010).

${ }^{19}$ Z. Lu and J. R. Dahn, J. Electrochem. Soc. 149, A815 (2002).

${ }^{20}$ A. D. Robertson and P. G. Bruce, Chem. Mater. 15, 1984 (2003).

${ }^{21}$ Y. Okamoto, J. Electrochem. Soc. 159, A152 (2012).

${ }^{22}$ D. Y. W. Yu and K. Yanagida, J. Electrochem. Soc. 158, A1015 (2011).

${ }^{23}$ M. M. Thackeray, S.-H. Kang, C. S. Johnson, J. T. Vaughey, R. Benedeka, and S. A. Hackney, J. Mater. Chem. 17, 3112 (2007).

${ }^{24}$ M. J. Lewis, B. D. Gaulin, L. Filion, C. Kallin, A. J. Berlinsky, H. A. Dabkowska, Y. Qiu, and J. R. D. Copley, Phys. Rev. B 72, 014408 (2005).

${ }^{25}$ J. Sugiyama, M. Månsson, Y. Ikedo, T. Goko, K. Mukai, D. Andreica, A. Amato, K. Ariyoshi, and T. Ohzuku, Phys. Rev. B 79, 184411 (2009).

${ }^{26}$ A. Olariu, P. Mendels, F. Bert, L. K. Alexander, A. V. Mahajan, A. D. Hillier, and A. Amato, Phys. Rev. B 79, 224401 (2009).

${ }^{27}$ A. Olariu, P. Mendels, F. Bert, B. G. Ueland, P. Schiffer, R. F. Berger, and R. J. Cava, Phys. Rev. Lett. 97, 167203 (2006).
${ }^{28}$ J. Sugiyama, H. Nozaki, J. H. Brewer, E. J. Ansaldo, G. D. Morris, and C. Delmas, Phys. Rev. B 72, 144424 (2005).

${ }^{29}$ K. Mukai, Y. Ikedo, H. Nozaki, J. Sugiyama, K. Nishiyama, D. Andreica, A. Amato, P. L. Russo, E. J. Ansaldo, J. H. Brewer et al., Phys. Rev. Lett. 99, 087601 (2007).

${ }^{30}$ J. Sugiyama, H. Itahara, J. H. Brewer, E. J. Ansaldo, T. Motohashi, M. Karppinen, and H. Yamauchi, Phys. Rev. B 67, 214420 (2003).

${ }^{31}$ J. Sugiyama, J. H. Brewer, E. J. Ansaldo, H. Itahara, T. Tani, M. Mikami, Y. Mori, T. Sasaki, S. Hébert, and A. Maignan, Phys. Rev. Lett. 92, 017602 (2004).

${ }^{32}$ J. Sugiyama, J. H. Brewer, E. J. Ansaldo, B. Hitti, M. Mikami, Y. Mori, and T. Sasaki, Phys. Rev. B 69, 214423 (2004).

${ }^{33}$ S. P. Bayrakci, C. Bernhard, D. P. Chen, B. Keimer, R. K. Kremer, P. Lemmens, C. T. Lin, C. Niedermayer, and J. Strempfer, Phys. Rev. B 69, 100410 (2004).

${ }^{34}$ P. Mendels, D. Bono, J. Bobroff, G. Collin, D. Colson, N. Blanchard, H. Alloul, I. Mukhamedshin, F. Bert, A. Amato et al., Phys. Rev. Lett. 94, 136403 (2005).

${ }^{35}$ G. Lang, J. Bobroff, H. Alloul, P. Mendels, N. Blanchard, and G. Collin, Phys. Rev. B 72, 094404 (2005).

${ }^{36}$ J. Sugiyama, H. Nozaki, Y. Ikedo, K. Mukai, J. H. Brewer, E. J. Ansaldo, G. D. Morris, D. Andreica, A. Amato, T. Fujii et al., Phys. Rev. Lett. 96, 037206 (2006).

${ }^{37}$ J. Sugiyama, Y. Ikedo, P. L. Russo, H. Nozaki, K. Mukai, D. Andreica, A. Amato, M. Blangero, and C. Delmas, Phys. Rev. B 76, 104412 (2007).

${ }^{38}$ J. Sugiyama, K. Mukai, Y. Ikedo, P. L. Russo, H. Nozaki, D. Andreica, A. Amato, K. Ariyoshi, and T. Ohzuku, Phys. Rev. B 78, 144412 (2008).

${ }^{39}$ J. Sugiyama, Y. Ikedo, K. Mukai, H. Nozaki, M. Månsson, O. Ofer, M. Harada, K. Kamazawa, Y. Miyake, J. H. Brewer et al., Phys. Rev. B 82, 224412 (2010).

${ }^{40}$ P. J. Baker, T. Lancaster, S. J. Blundell, M. L. Brooks, W. Hayes, D. Prabhakaran, and F. L. Pratt, Phys. Rev. B 72, 104414 (2005).

${ }^{41}$ J. Sugiyama, Y. Ikedo, O. Ofer, M. Månsson, E. J. Ansaldo, J. H. Brewer, K. H. Chow, H. Sakurai, and E. Takayama-Muromachi, J. Phys. Soc. Jpn. 78, 084715 (2009).

${ }^{42}$ K. Mukai, J. Sugiyama, Y. Ikedo, H. Nozaki, K. Kamazawa, D. Andreica, A. Amato, M. Månsson, J. H. Brewer, E. J. Ansaldo et al., J. Phys. Chem. C 114, 11320 (2010).

${ }^{43}$ I. Tomeno and M. Oguchi, J. Phys. Soc. Jpn. 67, 318 (1998).

${ }^{44}$ K. Nakamura, M. Yamamoto, K. Okamura, Y. Michihiro, I. Nakabayashi, and T. Kanashiro, Solid State Ionics 121, 301 (1999).

${ }^{45}$ P. Mustarelli, V. Massarotti, M. Bini, and D. Capsoni, Phys. Rev. B 55, 12018 (1997).

${ }^{46}$ K. Nakamura, H. Hirano, Y. Michihiro, and T. Moriga, Solid State Ionics 181, 1359 (2010). 
${ }^{47}$ J. Sugiyama, K. Mukai, Y. Ikedo, H. Nozaki, M. Månsson, and I. Watanabe, Phys. Rev. Lett. 103, 147601 (2009).

${ }^{48}$ T. Matsuzaki, K. Nishiyama, K. Nagamine, T. Yamazaki, M. Senba, J. M. Bailey, and J. H. Brewer, Phys. Lett. A 123, 91 (1989).

${ }^{49}$ R. S. Hayano, Y. J. Uemura, J. Imazato, N. Nishida, T. Yamazaki, and R. Kubo, Phys. Rev. B 20, 850 (1979).

${ }^{50}$ J. Sugiyama, ISIS experimental report No. 1120132.

${ }^{51}$ J. Sugiyama, H. Nozaki, M. Harada, K. Kamazawa, O. Ofer, M. Månsson, J. H. Brewer, E. J. Ansaldo, K. H. Chow, Y. Ikedo et al., Phys. Rev. B 84, 054430 (2011).

${ }^{52}$ P. J. Baker, I. Franke, F. L. Pratt, T. Lancaster, D. Prabhakaran, W. Hayes, and S. J. Blundell, Phys. Rev. B 84, 174403 (2011).

${ }^{53}$ J. Sugiyama, H. Nozaki, M. Harada, K. Kamazawa, Y. Ikedo, Y. Miyake, O. Ofer, M. Månsson, E. J. Ansaldo, K. H. Chow et al., Phys. Rev. B 85, 054111 (2012).

${ }^{54}$ G. M. Kalvius, D. R. Noakes, and O. Hartmann, Handbook on the Physics and Chemistry of Rare Earths (North-Holland, Amsterdam, 2001), Vol. 32, Chap. 206, pp. 55-451.

${ }^{55}$ W. B. Yelon, D. E. Cox, and M. Eibschütz, Phys. Rev. B 12, 5007 (1975).

${ }^{56}$ A. Taroni, S. T. Bramwell, and P. C. W. Holdsworth, J. Phys.: Condens. Matter 20, 275233 (2008).

${ }^{57}$ V. Massarotti, D. Capsoni, M. Bini, G. Chiodelli, C. B. Azzoni, M. C. Mozzati, and A. Paleari, J. Solid State Chem. 131, 94 (1997).

${ }^{58}$ K. Shimomura, K. Nishiyama, and R. Kadono, Phys. Rev. Lett. 89, 255505 (2002).

${ }^{59}$ K. Shimomura, R. Kadono, K. Ohishi, M. Mizuta, M. Saito, K. H. Chow, B. Hitti, and R. L. Lichti, Phys. Rev. Lett. 92, 135505 (2004).

${ }^{60}$ F. Damay, C. Martin, V. Hardy, A. Maignan, G. André, K. Knight, S. R. Giblin, and L. C. Chapon, Phys. Rev. B 81, 214405 (2010).
${ }^{61}$ K. M. Kojima, J. Yamanobe, H. Eisaki, S. Uchida, Y. Fudamoto, I. M. Gat, M. I. Larkin, A. Savici, Y. J. Uemura, P. P. Kyriakou et al., Phys. Rev. B 70, 094402 (2004).

${ }^{62}$ H. Nozaki, M. Janoschek, B. Roessli, J. Sugiyama, L. Keller, J. H. Brewer, E. J. Ansaldo, G. D. Morris, T. Takami, and H. Ikuta, Phys. Rev. B 76, 014402 (2007).

${ }^{63}$ P. L. Russo, J. Sugiyama, J. H. Brewer, E. J. Ansaldo, S. L. Stubbs, K. H. Chow, R. Jin, H. Sha, and J. Zhang, Phys. Rev. B 80, 104421 (2009).

${ }^{64}$ J. Sugiyama, Y. Ikedo, T. Goko, E. J. Ansaldo, J. H. Brewer, P. L. Russo, K. H. Chow, and H. Sakurai, Phys. Rev. B 78, 224406 (2008).

${ }^{65}$ O. Ofer, Y. Ikedo, T. Goko, M. Månsson, J. Sugiyama, E. J. Ansaldo, J. H. Brewer, K. H. Chow, and H. Sakurai, Phys. Rev. B 82, 094410 (2010).

${ }^{66}$ J. Sugiyama, M. Månsson, O. Ofer, K. Kamazawa, M. Harada, D. Andreica, A. Amato, J. H. Brewer, E. J. Ansaldo, H. Ohta et al., Phys. Rev. B 84, 184421 (2011).

${ }^{67}$ J. Sugiyama, H. Nozaki, M. Månsson, K. Prša, D. Andreica, A. Amato, M. Isobe, and Y. Ueda, Phys. Rev. B 85, 214407 (2012).

${ }^{68}$ C. Darie, P. Bordet, S. de Brion, M. Holzapfel, O. Isnard, A. Lecchi, J. E. Lorenzo, and E. Suard, Eur. Phys. J. B 43, 159 (2005).

${ }^{69}$ Y. Koyama, I. Tanaka, M. Nagao, and R. Kanno, J. Power Sources 189, 798 (2009).

${ }^{70}$ R. J. Borg and G. J. Dienes, An Introduction to Solid State Diffusion (Academic Press, San Diego, 1988), Chap. 3, pp. 53-77.

${ }^{71}$ P. Heitjans and S. Indris, J. Phys.: Condens. Matter 15, R1257 (2003).

${ }^{72}$ S. Levasseur, M. Ménétrier, Y. Shao-Horn, L. Gautier, A. Audemer, G. Demazeau, A. Largeteau, and C. Delmas, Chem. Mater. 15, 348 (2003).

${ }^{73}$ K. Momma and F. Izumi, J. Appl. Cryst. 41, 653 (2008). 